\title{
Article \\ Novel Series of Dual NRF2 Inducers and Selective MAO-B Inhibitors for the Treatment of Parkinson's Disease
}

\author{
Pablo Duarte ${ }^{1,2}$, Patrycja Michalska ${ }^{3}$, Enrique Crisman ${ }^{1,2,4}$, Antonio Cuadrado ${ }^{5}$ and Rafael León ${ }^{1, *(\mathbb{D})}$ \\ 1 Instituto de Química Médica, Consejo Superior de Investigaciones Científicas (IQM-CSIC), \\ 28006 Madrid, Spain; pablo.duarte@uam.es (P.D.); ecrisman@outlook.com (E.C.) \\ 2 Instituto Teófilo Hernando y Departamento de Farmacología y Terapéutica, Facultad de Medicina, \\ Universidad Autónoma de Madrid, 28029 Madrid, Spain \\ 3 Chemistry Department, Imperial College London, London W12 0BZ, UK; p.dziama@imperial.ac.uk \\ 4 Instituto de Investigación Sanitaria La Princesa (IIS-IS), Hospital Universitario de la Princesa, \\ 28006 Madrid, Spain \\ 5 Departamento de Bioquímica, Facultad de Medicina, Centro de Investigación Biomédica en Red Sobre \\ Enfermedades Neurodegenerativas (CIBERNED), Instituto de Investigación Sanitaria La Paz (IdiPaz), \\ Instituto de Investigaciones Biomédicas 'Alberto Sols' UAM-CSIC, Universidad Autónoma de Madrid, \\ 28029 Madrid, Spain; antonio.cuadrado@uam.es \\ * Correspondence: rafael.leon@iqm.csic.es
}

Citation: Duarte, P.; Michalska, P.; Crisman, E.; Cuadrado, A.; León, R. Novel Series of Dual NRF2 Inducers and Selective MAO-B Inhibitors for the Treatment of Parkinson's Disease. Antioxidants 2022, 11, 247. https:// doi.org/10.3390/antiox11020247

Academic Editor:

Alessandra Napolitano

Received: 31 December 2021

Accepted: 25 January 2022

Published: 27 January 2022

Publisher's Note: MDPI stays neutral with regard to jurisdictional claims in published maps and institutional affiliations.

Copyright: (C) 2022 by the authors. Licensee MDPI, Basel, Switzerland. This article is an open access article distributed under the terms and conditions of the Creative Commons Attribution (CC BY) license (https:// creativecommons.org/licenses/by/ $4.0 /)$.

\begin{abstract}
Parkinson's disease (PD) is the second most prevalent neurodegenerative disease. It is characterized by a complex network of physiopathological events where oxidative stress plays a central role among other factors such as neuroinflammation and protein homeostasis. Nuclear factor-erythroid 2 p45-related factor 2 (NRF2) has a multitarget profile itself as it controls a plethora of cellular processes involved in the progression of the disease. In this line, we designed a novel family of 2-(1H-indol-3-yl)ethan-1-amine derivatives as NRF2 inducers with complementary activities. Novel compounds are based on melatonin scaffold and include, among other properties, selective monoamine oxidase B (MAO-B) inhibition activity. Novel multitarget compounds exhibited NRF2 induction activity and MAO-B selective inhibition, combined with anti-inflammatory, antioxidant, and blood-brain barrier permeation properties. Furthermore, they exert neuroprotective properties against oxidative stress toxicity in PD-related in vitro. Hit compound $\mathbf{1 4}$ reduced oxidative stress markers and exerted neuroprotection in rat striatal slices exposed to 6-hydroxydopamine or rotenone. In conclusion, we developed a promising family of dual NRF2 inducers and selective MAO-B inhibitors that could serve as a novel therapeutic strategy for PD treatment.
\end{abstract}

Keywords: NRF2; MAO-B; Parkinson's disease; oxidative stress; neuroinflammation; multitarget

\section{Introduction}

Parkinson's disease (PD) is the second most prevalent neurodegenerative disease, affecting $2-3 \%$ population aged 65 years or over [1]. PD is characterized by a progressive loss of dopaminergic neurons in the substantia nigra pars compacta (SNpc), dopamine levels reduction and the presence of $\alpha$-synuclein protein deposits known as Lewy bodies [1-4]. Although PD etiology remains unknown, evidence indicates that oxidative stress (OS) plays an essential role in its onset and development, associated with other pathological pathways, including $\alpha$-synuclein proteostasis, mitochondrial dysfunction, calcium dyshomeostasis, axonal transport dysfunction, and neuroinflammation. Crosstalk between these factors induces increased OS, creating a feedback loop that accelerates neurodegeneration $[1,5]$. Importantly, current PD therapies are merely symptomatic, highlighting the need for effective treatments able to modify disease progression.

Currently, PD treatments include monoamine oxidase (MAO) inhibitors (selegiline, rasagiline, and safinamide [6]) directed to increasing dopamine levels at the synaptic clef 
and to reducing OS generated during dopamine metabolism [7]. MAO enzymes (MAO$\mathrm{A}$ and B isozymes) catalyze the oxidative deamination of biological amines including dopamine. Amine metabolism produces high quantities of hydrogen peroxide that decomposes to generate free radicals, thus contributing to an exacerbated OS status that, finally, induces high cytotoxicity under pathological conditions [6]. Additionally, the use of selective MAO-B inhibitors for PD is based on several observations. Human brain postmortem studies have demonstrated a MAO-B levels increase with aging, the main risk factor of PD [8]. Given that MAO-B is mainly present in glial cells, its levels are also augmented as a consequence of gliosis [9]. Thus, astrocytes surrounding dopaminergic neurons at SNpc contribute to the increased OS status due to dopamine metabolism. As previously stated, hydrogen peroxide is produced in this process and it can be transferred to close neurons, which usually contain lower glutathione (GSH) levels than astrocytes to control OS. it makes them more susceptible to this toxic stimulus $[10,11]$. Additionally, MAO-B is responsible for $\gamma$-aminobutyric acid (GABA) synthesis [12]. Released GABA from astrocytes inhibits adjacent dopaminergic neurons at substantia nigra further decreasing dopamine levels $[13,14]$. Therefore, although currently used MAO-B inhibitors only offer symptomatic relief being unable to modify the progression of the disease, these recent findings support the therapeutic potential of selective MAO-B inhibition.

Regarding the multifactorial nature of PD and its complex pathological network, we considered the use of the intrinsic antioxidant and anti-inflammatory response as a key target for its treatment. In this line, the nuclear factor-erythroid 2 p45-related factor 2 (NRF2) is considered the master regulator of the phase II antioxidant response. It is a transcription factor encoded by NFE2L2 gene that controls about $1 \%$ of human genes preceded by the antioxidant response element (ARE) [15]. It regulates a plethora of processes including inflammation, redox balance, and protein homeostasis [16,17]. Importantly, NRF2 activity decreases during aging the main PD risk factor. Moreover, it has been widely demonstrated that the expression of several NRF2-dependent genes is increased in PD brains (i.e., heme oxygenase 1 (HMOX1), glutamate-cysteine ligase regulatory subunit (GCLM), sequestosome-1 (SQSTM), and NAD(P)H:quinone oxidoreductase 1 (NQO1)) [18-22]. This phase II response activation under pathological conditions is an attempt to respond to exacerbated OS. Additionally, NRF2 exhibits nuclear localization in nigral neurons from postmortem samples of PD patients [23] and a positive correlation between its protein levels in cerebral spinal fluid of PD patients and the disease duration or motor scores [24]. Interestingly, in these post-mortem samples, NRF2-dependent proteins NQO1 and p62 were partially trapped in Lewy bodies, suggesting an impaired capacity of the NRF" signature to resolve increased OS status [22]. In addition, there is an association between a protective NFE2L2 promoter single nucleotide polymorphisms (SNPs) haplotype with a delayed onset and reduced PD risk $[21,22,25,26]$. Complementing this clinical evidence, NRF2 therapeutic potential for PD treatment is further supported by numerous in vitro and in vivo studies in which NRF2-ARE pathway activation demonstrates protective role in $\alpha$-synucleinopathy PD models. In this line, ventral midbrain injection of adeno-associated viral vector (rAAV) to express human $\alpha$-synuclein in NFE2L2 $\left({ }^{-/-}\right)$-deficient mice induced exacerbated protein aggregation, neuroinflammation, and neuronal death [27]. Moreover, astrocyte-specific NFE2L2 overexpression significantly reduced synuclein accumulation and provided neuroprotection in the $\alpha$-synuclein mutant (A53T) mouse model [28]. Interestingly, NRF2 pharmacological activation by dimethyl fumarate (DMF), an approved drug for the treatment of relapsing-remitting multiple sclerosis, reduced dopaminergic neuron loss in the substantia nigra, and decreased astrocytosis and microgliosis in a $\alpha$-synuclein mouse model [22]. DMF also showed neuroprotection against 6-hydroxydopamine (6-OHDA) [29] and methyl-4-phenyl-1,2,3,6-tetrahydropyridine (MPTP) in in vivo mouse models [30-32], being able to modulate microglial dynamics and neuroinflammation [21,33].

Regarding its regulation, under basal conditions cytosolic NRF2 is bound to its repressor protein Kelch like ECH associated protein 1 (KEAP1) that promoted its proteasomal degradation. However, under OS conditions or in the presence of electrophilic compounds, 
KEAP1 repressor activity is weakened, leading to NRF2 nuclear accumulation [34]. Electrophilic compounds are able to react with key cysteine residues at KEAP1 inducing conformational changes that prevent NRF2 degradation $[35,36]$. A prominent example of this process is the DMF mechanism of action whose derived metabolite, monomethyl fumarate (MMF), reacts with KEAP1-Cys151 to induce the NRF2-ARE pathway activation [37].

Considering multitarget design, melatonin is a neurohormone with a pleiotropic profile, with remarkable antioxidant properties, able to scavenge free radicals generating several metabolites that can also trap more free radicals. This capacity is known as the antioxidant cascade of melatonin [38]. Moreover, it enhances the expression of antioxidant and downregulates pro-oxidant enzymes [39], leading to an interesting neuroprotective profile [40]. Melatonin has shown protective effects in several PD animal models based on brain injections of 6-OHDA [41-43], MPTP [44-47], rotenone [48,49], or lentiviral vectors encoding mutant human $\alpha$-synuclein [50]. Clinical trials conducted for PD treatment with melatonin mainly showed effectiveness in controlling sleep disturbances associated with the disease and significant reductions in the unified Parkinson's disease rating scale (UPDRS) score in certain cases [51-57]. However, additional clinical studies are needed with doses that allometrically projected to humans are at least 10-times higher than those used in clinics [58].

In view of previously introduced evidences, we hypothesized that the combination of NRF2 inducing activity with selective MAO-B inhibition could result in a beneficial therapeutic approach for PD treatment. To this end, we developed a new family of multitarget compounds considering melatonin structure as a scaffold, given its interesting neuroprotective profile. Structural modifications led to the inclusion of NRF2-ARE pathway induction capacity and, finally, novel compounds were also designed as selective MAO-B inhibitors based on structural similarity with known inhibitors. Here, we described one of the first series of dual NRF2 activators and selective MAO-B inhibitors, as an innovative approach for the development of new PD therapies.

\section{Materials and Methods}

\subsection{Chemistry}

\subsubsection{Chemical Methods}

All reagents were commercial compounds with high purity. Argon atmosphere was employed for all reactions. Triethylamine $\left(\mathrm{Et}_{3} \mathrm{~N}\right)$ and dichloromethane $(\mathrm{DCM})$ were dried by distillation over $\mathrm{NaOH}$ and $\mathrm{CaH}_{2}$, respectively. Acetonitrile $(\mathrm{MeCN})$ was purchased anhydrous. Analytical thin layer chromatography (TLC) was carried out on aluminum plates with Merck Silicagel 60 F254 and visualized by UV irradiation $(254 \mathrm{~nm})$. Flash column chromatography was performed with Merck Kieselgel 60 (230-400 mesh). Proton nuclear magnetic resonance $\left({ }^{1} \mathrm{H}\right.$ NMR) spectra were recorded in DMSO at room temperature in a Bruker DRX-300 MHz device. The proton spectra was reported as follows: chemical shifts $(\delta)$ in ppm (number of protons, multiplicity, coupling constant $\mathrm{J}$ in $\mathrm{Hz}$, assignment). Carbon nuclear magnetic resonance $\left({ }^{13} \mathrm{C}\right.$ NMR) were recorded in DMSO-d6 at room temperature using the same spectrometers at $75 \mathrm{MHz}$. The infrared spectra (IR) were obtained using an Agilent Technologies Cary 630 FTIR spectrophotometer from a thin film. Purity of the compounds was studied by high-performance liquid chromatography (HPLC), coupled with high-resolution mass spectrometry (HRMS) electrospray with positive mode detection for mass determination, using a mass spectrometer with a quadrupole time-of-fight analyzer (QTOF) model QSTAR pulsar I (Applied Biosystems, Waltham, MA, USA).

\subsubsection{Preparation of Tert-butyl-(2-(5-hydroxy- $1 H$-indol-3-yl)ethyl)carbamate (3)}

To a solution of serotonin hydrochloride (1.00 eq) in tetrahydrofuran (THF) under argon, di-tert-butyl dicarbonate $(1.10 \mathrm{eq})$ and $\mathrm{Et}_{3} \mathrm{~N}(1.10 \mathrm{eq})$ were added slowly. The solution was stirred under argon for $90 \mathrm{~min}$, quenched with $\mathrm{NH}_{4} \mathrm{Cl}$, and extracted with ethyl acetate $(\mathrm{AcOEt})$. The organic layers were dried over $\mathrm{MgSO}_{4}$ and concentrated under vacuum. The resulting product was purified by flash chromatography on silica gel (Hexane:AcOEt 
$0-45 \%)$ to afford compound 3 as a white solid (99\% yield). Experimental results were in agreement with previously reported data [1].

2.1.3. General Procedure for the Synthesis of

3-(2-((Tert-butoxycarbonyl)amino)ethyl)-1H-indol-5-yl Cinnamate Derivatives $(6,7)$

To a solution of tert-butyl-(2-(5-hydroxy-1H-indol-3-yl)ethyl)carbamate (3, 1.00 eq) and the corresponding acrylic acid derivative (4 or 5, 1.20 eq) in DCM under argon, $N, N^{\prime}$ dicyclohexylcarbodiimide (DCC, 1.20-1.50 eq), $\mathrm{HOBt},(0.20 \mathrm{eq})$, and $\mathrm{Et}_{3} \mathrm{~N}$ (1.50 eq) were added. The resulting solution was stirred at room temperature. When completed, the reaction was quenched with distilled water and stirred for $15 \mathrm{~min}$. Then, the mixture was extracted three times with DCM, and washed with $10 \%$ aqueous $\mathrm{NaHCO}_{3}$ and a saturated solution of $\mathrm{NH}_{4} \mathrm{Cl}$. The organic layers were dried over $\mathrm{MgSO}_{4}$ and filtered. The resulting product was purified by flash chromatography on silica gel using DCM:MeOH mixtures $(0-3 \%)$ or hexane:AcOEt mixtures $(0-80 \%)$ as eluent to afford the corresponding 3-(2-((tert-butoxycarbonyl)amino)ethyl)- $1 H$-indol-5-yl cinnamate derivative.

2.1.4. General Procedure for the Synthesis of 3-(2-Aminoethyl)- $1 H$-indol-5-yl Cinnamate Derivatives $(\mathbf{8}, \mathbf{9})$

To a solution of the corresponding 3-(2-((tert-butoxycarbonyl)amino)ethyl)-1H-indol5 -yl cinnamate derivative ( 6 or 7, 1.00 eq) in DCM under argon, trifluoroacetic acid (TFA, $1.05 \mathrm{eq}$ ) was added. The resulting solution was stirred at room temperature. When completed, the reaction was quenched with $\mathrm{NaHCO}_{3}$, then extracted with $\mathrm{AcOEt}$ and washed with a saturated solution of $\mathrm{NH}_{4} \mathrm{Cl}$. The organic layers were dried over $\mathrm{MgSO}_{4}$ and filtered to yield the corresponding 3-(2-aminoethyl)- $1 \mathrm{H}$-indol-5-yl cinnamate derivative.

\subsubsection{General Procedure for the Synthesis of Amine Derivatives 10-15}

To a solution of the appropriate 5-methoxytryptamine derivative (1.00 eq) in $\mathrm{MeCN}$, propargyl bromide (1.00 eq) was added. $\mathrm{Et}_{3} \mathrm{~N}$ (1.00 eq) was added in the case of a low reaction rate. The resulting solution was stirred at room temperature upon completion. Once completed, products were purified by flash chromatography on silica gel using $\mathrm{DCM} / \mathrm{MeOH}$ mixtures $0-5 \%$ or petroleum ether/EtOAc; $\mathrm{MeOH} 0-100 \% ; 0-2.5 \%$ as eluent, to yield the desired product.

\subsubsection{General Procedure for the Synthesis of Amide Derivatives 16-18}

To a solution of DCC (1.15 eq) in dry DCM under argon, 2-propynoic acid (1.15 eq) and the appropriate 5-methoxytryptamine derivative (1.00 eq) were added. The resulting solution was stirred at room temperature. Once completed, the solution was filtered and the solvent was removed by evaporation at low pressure. The resulting products were purified by flash chromatography on silica gel using petroleum ether/EtOAc $0-100 \%$ or petroleum ether/DCM; $\mathrm{MeOH} 0-100 \% ; 0-2.5 \%$ to yield the corresponding amide product.

\subsubsection{3-(2-((Tert-butoxycarbonyl)amino)ethyl)-1H-indol-5-yl Cinnamate (6)}

General procedure 2.1.3, tert-butyl-(2-(5-hydroxy-1H-indol-3-yl)ethyl)carbamate (3, $1.0 \mathrm{~g}, 3.62 \mathrm{mmol})$ and cinnamic acid $(4,805 \mathrm{mg}, 5.43 \mathrm{mmol}), \mathrm{DCC}(1.12 \mathrm{~g}, 5.43 \mathrm{mmol}), \mathrm{HOBt}$ ( $98 \mathrm{mg}, 0.72 \mathrm{mmol}), \mathrm{Et}_{3} \mathrm{~N}(700 \mu \mathrm{L}, 5.43 \mathrm{mmol})$, and DCM $(20 \mathrm{~mL}), 16 \mathrm{~h}$, flash chromatography on silica gel (hexane: EtOAc 0-50\%) to afford compound $\mathbf{6}$ as a white solid $(1.17 \mathrm{~g}, 80 \%$ yield). $\lambda_{\max }\left(\right.$ film) $/ \mathrm{cm}^{-1} 3377,3301,3060,2978,2936,1725,1635,1580,1388,1171,1142,1102$, 982, 873, 763. ${ }^{1} \mathrm{H}$ NMR $(300 \mathrm{MHz}, \mathrm{DMSO}) \delta_{\mathrm{H}} 10.94\left(1 \mathrm{H}, \mathrm{s}_{\mathrm{br}}, \mathrm{NH}\right), 7.87(1 \mathrm{H}, \mathrm{d}, \mathrm{J}=16.1 \mathrm{~Hz}$, $\left.3^{\prime}-\mathrm{H}\right), 7.84-7.80\left(2 \mathrm{H}, \mathrm{m}, 2^{\prime \prime}-\mathrm{H}\right), 7.48-7.44\left(3 \mathrm{H}, \mathrm{m}, 32^{\prime \prime}-\mathrm{H}, 42^{\prime \prime}-\mathrm{H}\right), 7.37(1 \mathrm{H}, \mathrm{d}, \mathrm{J}=8.6 \mathrm{~Hz}, 7-\mathrm{H})$, $7.29(1 \mathrm{H}, \mathrm{d}, \mathrm{J}=2.2 \mathrm{~Hz}, 4-\mathrm{H}), 7.22(1 \mathrm{H}, \mathrm{d}, \mathrm{J}=2.3 \mathrm{~Hz}, 2-\mathrm{H}), 6.93-6.88\left(2 \mathrm{H}, \mathrm{m}, 2^{\prime}-\mathrm{H}, 6-\mathrm{H}\right), 3.24-$ $\left.3.15\left(2 \mathrm{H}, \mathrm{m}, \mathrm{CH}_{2} \mathrm{CH}_{2} \mathrm{NHCOO}\left(\mathrm{CH}_{3}\right)_{3}\right), 2.79\left(2 \mathrm{H}, \mathrm{t}, \mathrm{J}=7.4 \mathrm{~Hz}, \mathrm{CH}_{2} \mathrm{CH}_{2} \mathrm{NHCOO} \mathrm{CH}_{3}\right)_{3}\right)$, $1.38\left(9 \mathrm{H}, \mathrm{S}, \mathrm{CH}_{2} \mathrm{CH}_{2} \mathrm{NHCOO}\left(\mathrm{CH}_{3}\right)_{3}\right) .{ }^{13} \mathrm{C} \mathrm{NMR}(75 \mathrm{MHz}, \mathrm{DMSO}) \delta_{\mathrm{C}} 165.7,155.5,145.7$, $145.5,143.2,133.9,130.6,128.9,128.5,127.4,124.2,117.7,115.3,112.2,111.6,110.3,77.4$, 40.8, 28.2, 25.3. HRMS (ES ${ }^{+}$) mass calculated for $\mathrm{C}_{24} \mathrm{H}_{26} \mathrm{~N}_{2} \mathrm{O}_{6} 406.1893$; found $\left[(\mathrm{M}+\mathrm{H})^{+}\right]$ 407.1965; found $\left[(\mathrm{M}+\mathrm{Na})^{+}\right] 429.1917$. 
2.1.8. (E)-3-(2-((Tert-butoxycarbonyl)amino)ethyl)-1H-indol-5-yl 3-p-tolylacrylate (7)

General procedure 2.1.3, tert-butyl-(2-(5-hydroxy- $1 H$-indol-3-yl)ethyl)carbamate (3, $1.0 \mathrm{~g}, 3.62 \mathrm{mmol}$ ) and (E)-3-( $p$-tolyl)acrylic acid $(5,880 \mathrm{mg}, 5.43 \mathrm{mmol}), \mathrm{DCC}(1.12 \mathrm{~g}, 5.43$ $\mathrm{mmol}), \mathrm{HOBt}(98 \mathrm{mg}, 0.72 \mathrm{mmol}), \mathrm{Et}_{3} \mathrm{~N}(700 \mu \mathrm{L}, 5.43 \mathrm{mmol})$ and DCM $(20 \mathrm{~mL}), 16 \mathrm{~h}$, flash chromatography on silica gel (Hexane:EtOAc $0-50 \%$ ) to afford 7 as a white solid (1.18 g, $78 \%$ yield). $\lambda_{\max }$ (film) $/ \mathrm{cm}^{-1} 3374,3306,2977,2930,2861,1720,1690,1516,1455,1368,1253$, $1169,991,815 .{ }^{1} \mathrm{H}$ NMR $(300 \mathrm{MHz}, \mathrm{DMSO}) \delta_{\mathrm{H}} 10.93\left(1 \mathrm{H}, \mathrm{s}_{\mathrm{br}}, \mathrm{NH}\right), 7.83(1 \mathrm{H}, \mathrm{d}, \mathrm{J}=15.9 \mathrm{~Hz}$, $\left.3^{\prime}-\mathrm{H}\right), 7.70\left(2 \mathrm{H}, \mathrm{d}, \mathrm{J}=7.9 \mathrm{~Hz}, 32^{\prime \prime}-\mathrm{H}\right), 7.37(1 \mathrm{H}, \mathrm{d}, \mathrm{J}=8.6 \mathrm{~Hz}, 7-\mathrm{H}), 7.29-7.27(3 \mathrm{H}, \mathrm{m}, 4-\mathrm{H}$, $\left.22^{\prime \prime}-\mathrm{H}\right), 7.22(1 \mathrm{H}, \mathrm{d}, \mathrm{J}=2.3 \mathrm{~Hz}, 2-\mathrm{H}), 6.91-6.80\left(3 \mathrm{H}, \mathrm{m}, 6-\mathrm{H}, 2^{\prime}-\mathrm{H}, \mathrm{NH}\right), 3.22-3.16(2 \mathrm{H}, \mathrm{m}$, $\left.\mathrm{CH}_{2} \mathrm{CH}_{2} \mathrm{NHCOO}\left(\mathrm{CH}_{3}\right)_{3}\right), 2.79\left(2 \mathrm{H}, \mathrm{t}, \mathrm{J}=7.4 \mathrm{~Hz}, \mathrm{CH}_{2} \mathrm{CH}_{2} \mathrm{NHCOO}\left(\mathrm{CH}_{3}\right)_{3}\right), 2.36(3 \mathrm{H}, \mathrm{s}$, $\left.\mathrm{CH}_{3}-\mathrm{Ph}\right) 1.38\left(9 \mathrm{H}, \mathrm{s}, \mathrm{CH}_{2} \mathrm{CH}_{2} \mathrm{NHCOO}\left(\mathrm{CH}_{3}\right)_{3}\right) .{ }^{13} \mathrm{C}$ NMR $\left(75 \mathrm{MHz}, \mathrm{DMSO}-\mathrm{d}_{6}\right) \delta_{\mathrm{C}} 165.8$, $155.5,145.7,143.2,140.7,133.9,131.2,129.5,128.5,127.4,124.1,116.5,115.3,112.2$, 111.6, 110.3, 77.3, 40.8, 28.2, 25.3, 21.0. HRMS (ES ${ }^{+}$) mass calculated for $\mathrm{C}_{25} \mathrm{H}_{28} \mathrm{~N}_{2} \mathrm{O}_{4} 420.2049$; found $\left[(\mathrm{M}+\mathrm{H})^{+}\right]$421.2126; found $\left[(\mathrm{M}+\mathrm{Na})^{+}\right] 443.1931$.

\subsubsection{3-(2-Aminoethyl)-1H-indol-5-yl Cinnamate (8)}

General procedure 2.1.4, 3-(2-((tert-butoxycarbonyl)amino)ethyl)- $1 H$-indol-5-yl cinnamate $(6,300 \mathrm{mg}, 0.738 \mathrm{mmol})$, TFA $(59.0 \mu \mathrm{L}, 0.775 \mathrm{mmol})$ in dry DCM $(10 \mathrm{~mL}), 24 \mathrm{~h}$ to afford compound 8 as a yellow-orange solid $\left(60 \%\right.$ yield)). ${ }^{1} \mathrm{H}$ NMR $(300 \mathrm{MHz}, \mathrm{DMSO}) \delta_{\mathrm{H}} 11.03$ $\left(1 \mathrm{H}, \mathrm{sbr}_{\mathrm{br}} \mathrm{NH}\right), 7.90-7.78\left(3 \mathrm{H}, \mathrm{m}, 3^{\prime}-\mathrm{H}, 22^{\prime \prime}-\mathrm{H}\right), 7.47\left(3 \mathrm{H}, \mathrm{m}, 32^{\prime \prime}-\mathrm{H}, 42^{\prime \prime}-\mathrm{H}\right), 7.41-7.33(2 \mathrm{H}, \mathrm{m}$, 7-H, 4-H), 7.27 (1H, s, 2-H), 6.94-6.87 (2H, m, 2'-H, 6-H), 3.02-2.91 (2H, m, $\left.\mathrm{CH}_{2} \mathrm{CH}_{2} \mathrm{NH}_{2}\right)$, 2.91-2.82 (2H, m, $\left.\mathrm{CH}_{2} \mathrm{CH}_{2} \mathrm{NH}_{2}\right), 1.87\left(2 \mathrm{H}, \mathrm{d}, \mathrm{J}=4.9 \mathrm{~Hz}, \mathrm{NH}_{2}\right) .{ }^{13} \mathrm{C} \mathrm{NMR}(75 \mathrm{MHz}, \mathrm{DMSO})$ $\delta_{C} 168.4,148.5,146.0,136.8,136.6,133.4,131.66,131.2,129.8,127.4,120.33,118.1,114.5,113.6$, 113.1, 28.1, 24.3. HPLC-HRMS (ES+) calculated mass for $\mathrm{C}_{19} \mathrm{H}_{18} \mathrm{~N}_{2} \mathrm{O}_{2} 306.1368$; found [(M $\left.+\mathrm{H})^{+}\right]$307.1440; found $\left[(\mathrm{M}+2 \mathrm{H})^{+}\right]$308.1473; found $\left[(2 \mathrm{M}+\mathrm{H})^{+}\right]$613.2801.

\subsubsection{3-(2-Aminoethyl)-1H-indol-5-yl (E)-3-(p-tolyl)acrylate (9)}

General procedure 2.1.4, (3-(2-((tert-butoxycarbonyl)amino)ethyl)-1H-indol-5-yl (E)-3(p-tolyl)acrylate $(7,300 \mathrm{mg}, 0.714 \mathrm{mmol})$, TFA $(57.0 \mu \mathrm{L}, 0.750 \mathrm{mmol})$ in dry DCM $(10 \mathrm{~mL})$, $24 \mathrm{~h}$ to afford compound 9 as a yellow solid (90\% yield). ${ }^{1} \mathrm{H}$ NMR (300 MHz, DMSO) $\delta_{\mathrm{H}}$ $11.08\left(1 \mathrm{H}, \mathrm{s}_{\mathrm{br}}, \mathrm{NH}\right), 7.82\left(1 \mathrm{H}, \mathrm{d}, \mathrm{J}=15.9 \mathrm{~Hz}, 3^{\prime}-\mathrm{H}\right), 7.70\left(2 \mathrm{H}, \mathrm{d}, \mathrm{J}=8.2 \mathrm{~Hz}, 22^{\prime \prime}-\mathrm{H}\right), 7.43-7.33$ $(2 \mathrm{H}, \mathrm{m}, 7-\mathrm{H}, 4-\mathrm{H}), 7.32-7.24\left(3 \mathrm{H}, \mathrm{m}, 3{ }^{\prime \prime} \mathrm{H}, 2-\mathrm{H}\right), 6.91(1 \mathrm{H}, \mathrm{dd}, \mathrm{J}=8.6, \mathrm{~J}=2.4 \mathrm{~Hz}, 6-\mathrm{H}), 6.83$ $\left(1 \mathrm{H}, \mathrm{d}, \mathrm{J}=16.0 \mathrm{~Hz}, 2^{\prime}-\mathrm{H}\right), 3.32\left(2 \mathrm{H}, \mathrm{s}_{\mathrm{br}}, \mathrm{NH}_{2}\right), 3.05\left(2 \mathrm{H}, \mathrm{t}, \mathrm{J}=7.6 \mathrm{~Hz}, \mathrm{CH}_{2} \mathrm{CH}_{2} \mathrm{NH}_{2}\right), 2.94$ $\left(2 \mathrm{H}, \mathrm{t}, \mathrm{J}=7.7 \mathrm{~Hz}, \mathrm{CH}_{2} \mathrm{CH}_{2} \mathrm{NH}_{2}\right), 2.36\left(3 \mathrm{H}, \mathrm{s}, \mathrm{Ph}_{-} \mathrm{CH}_{3}\right) ;{ }^{13} \mathrm{C} \mathrm{NMR}(75 \mathrm{MHz}, \mathrm{DMSO}) \delta_{\mathrm{C}} 168.5$, 148.6, 146.1, 143.5, 136.8, 134.0, 132.3, 131.3, 129.6, 127.6, 119.1, 118.3, 114.6, 113.0, 112.5, 42.4, 26.2, 23.8. HPLC-HRMS $\left(\mathrm{ES}^{+}\right)$calculated mass for $\mathrm{C}_{20} \mathrm{H}_{20} \mathrm{~N}_{2} \mathrm{O}_{2} 320.1525$; found $\left[(\mathrm{M}+\mathrm{H})^{+}\right]$ 321.1606; found $\left[(\mathrm{M}+2 \mathrm{H})^{+}\right] 322.1613$.

\subsubsection{N-(2-(5-Methoxy-1H-indol-3-yl)ethyl)prop-2-yn-1-amine (10)}

General procedure 2.1.5, 5-methoxytryptamine ( $50 \mathrm{mg}, 0.263 \mathrm{mmol})$ in $\mathrm{MeCN}(8 \mathrm{~mL})$, propargyl bromide $(29.3 \mu \mathrm{L}, 0.263 \mathrm{mmol}), 18 \mathrm{~h}$, flash chromatography on silica gel (DCM:MeOH $0-3 \%$ ) to afford compound 10 as a red oil (70\% yield). $\lambda_{\max }(\mathrm{film}) / \mathrm{cm}^{-1} 3413,3279,2919,2829$, $1587,1483,1216,797,643 .{ }^{1} \mathrm{H}$ NMR $(300 \mathrm{MHz}, \mathrm{DMSO}) \delta_{\mathrm{H}} 10.61\left(1 \mathrm{H}, \mathrm{s}_{\mathrm{br}}, \mathrm{NH}\right), 7.22(1 \mathrm{H}, \mathrm{d}, \mathrm{J}=$ $8.7 \mathrm{~Hz}, \mathrm{~d}, 7-\mathrm{H}), 7.09(1 \mathrm{H}, \mathrm{d}, \mathrm{J}=2.3 \mathrm{~Hz}, 2-\mathrm{H}), 7.00(1 \mathrm{H}, \mathrm{d}, \mathrm{J}=2.3 \mathrm{~Hz}, 4-\mathrm{H}), 6.71(1 \mathrm{H}, \mathrm{dd}, \mathrm{J}=8.7, \mathrm{~J}=$ $2.4 \mathrm{~Hz}, 6-\mathrm{H}), 3.76\left(3 \mathrm{H}, \mathrm{s}, \mathrm{O}-\mathrm{CH}_{3}\right), 3.41-3.27\left(3 \mathrm{H}, \mathrm{m}, \mathrm{CH}_{2} \mathrm{CCH}, \mathrm{CH}_{2} \mathrm{CH}_{2} \mathrm{NH}\right), 3.04(1 \mathrm{H}, \mathrm{t}, \mathrm{J}=2.3$, $\left.\mathrm{CH}_{2} \mathrm{CCH}\right), 2.88-2.73\left(4 \mathrm{H}, \mathrm{m}, \mathrm{CH}_{2} \mathrm{CH}_{2} \mathrm{NH}, \mathrm{CH}_{2} \mathrm{CH}_{2} \mathrm{NH}\right) ;{ }^{13} \mathrm{C}$ NMR $(75 \mathrm{MHz}, \mathrm{DMSO}) \delta_{\mathrm{C}} 152.8$, 131.3, 127.5, 123.2, 112.2, 111.9, 110.9, 100.1, 83.1, 73.4, 55.3, 48.6, 37.3, 25.1. HPLC-HRMS (ES ${ }^{+}$) calculated mass for $\mathrm{C}_{14} \mathrm{H}_{16} \mathrm{~N}_{2} \mathrm{O} 228.1273$; found $\left[(\mathrm{M}+\mathrm{H})^{+}\right]$229.1325; purity $96 \%$ (HPLC).

\subsubsection{3-(2-(Prop-2-yn-1-ylamino)ethyl)-1H-indol-5-yl Cinnamate (11)}

General procedure 2.1.5, 3-(2-aminoethyl)-1 $H$-indol-5-yl cinnamate $(107 \mathrm{mg}, 0.349 \mathrm{mmol})$ in $\mathrm{MeCN}(10 \mathrm{~mL})$, propargyl bromide $(51.9 \mu \mathrm{L}, 0.349 \mathrm{mmol})$ and $\mathrm{Et}_{3} \mathrm{~N}(46.3 \mu \mathrm{L}, 0.349 \mathrm{mmol})$ were added, $72 \mathrm{~h}$, flash chromatography on silica gel (petroleum ether:EtOAc 0-100\%:MeOH $0-3 \%$ ) to afford compound 11 as a yellow solid (31\% yield). $\lambda_{\max }\left(\right.$ film) $/ \mathrm{cm}^{-1} 3434,3257$, 
2918, 1626, 1472, 1388, 1299, 1212, 1038, 971. ${ }^{1} \mathrm{H}$ NMR (300 MHz, DMSO) $\delta_{\mathrm{H}} 8.40(1 \mathrm{H}, \mathrm{d}$, $\mathrm{J}=9.0 \mathrm{~Hz}, 7-\mathrm{H}), 8.24(1 \mathrm{H}, \mathrm{s}, 2-\mathrm{H}), 7.99-7.88\left(6 \mathrm{H}, \mathrm{m}, 22^{\prime \prime}-\mathrm{H}, 3^{\prime}-\mathrm{H}, \mathrm{NH}, \mathrm{NH}_{2}{ }^{+}\right), 7.73(1 \mathrm{H}, \mathrm{d}$, $\left.\mathrm{J}=15.4 \mathrm{~Hz}, 2^{\prime}-\mathrm{H}\right), 7.53-7.47\left(3 \mathrm{H}, \mathrm{m}, 32^{\prime \prime}-\mathrm{H}, 42^{\prime \prime}-\mathrm{H}\right), 7.27(1 \mathrm{H}, \mathrm{d}, \mathrm{J}=2.5 \mathrm{~Hz}, 4-\mathrm{H}), 7.05(1 \mathrm{H}, \mathrm{dd}$, $\mathrm{J}=8.9, \mathrm{~J}=2.6 \mathrm{~Hz}, 6-\mathrm{H}), 4.88\left(2 \mathrm{H}, \mathrm{d}, \mathrm{J}=2.4 \mathrm{~Hz}, \mathrm{CH}_{2} \mathrm{CCH}\right), 3.57\left(1 \mathrm{H}, \mathrm{t}, \mathrm{J}=2.4 \mathrm{~Hz}, \mathrm{CH}_{2} \mathrm{CCH}\right)$, 3.24-3.16 (2H, m, 2H, $\left.\mathrm{CH}_{2} \mathrm{CH}_{2} \mathrm{NH}\right), 3.00\left(2 \mathrm{H}, \mathrm{t}, \mathrm{J}=7.5 \mathrm{~Hz}, \mathrm{CH}_{2} \mathrm{CH}_{2} \mathrm{NH}\right) .{ }^{13} \mathrm{C} \mathrm{NMR}(75 \mathrm{MHz}$, DMSO) $\delta_{\mathrm{C}} 163.9,154.5,146.2,134.8,131.6,131.2,131.1,129.4,129.3,125.4,118.2,117.7,117.4$, 114.1, 104.2, 79.8, 78.6, 56.4, 38.6, 23.2. HPLC-HRMS $\left(\mathrm{ES}^{+}\right)$calculated mass for $\mathrm{C}_{22} \mathrm{H}_{20} \mathrm{~N}_{2} \mathrm{O}_{2}$ 344.1525; found $\left[(\mathrm{M}+\mathrm{H})^{+}\right]$345.1595; $\left[(2 \mathrm{M}+\mathrm{H})^{+}\right]$689.3136; purity 96\% (HPLC).

\subsubsection{3-(2-(Prop-2-yn-1-ylamino)ethyl)-1H-indol-5-yl-(E)-3-(p-tolyl)acrylate (12)}

General procedure 2.1.5, 3-(2-aminoethyl)-1H-indol-5-yl-(E)-3-( $p$-tolyl) acrylate (95.0 mg, $0.296 \mathrm{mmol})$ in $\mathrm{MeCN}(10 \mathrm{~mL})$, propargyl bromide $(92.9 \mu \mathrm{L}, 0.296 \mathrm{mmol})$ and $\mathrm{Et}_{3} \mathrm{~N}(40 \mu \mathrm{L}$, $0.296 \mathrm{mmol}$ ) were added, $72 \mathrm{~h}$, flash chromatography on silica gel (petroleum ether:EtOAc $0-100 \%: \mathrm{MeOH} 0-3 \%$ ) to afford compound 12 as a yellow solid (30\% yield). $\lambda_{\max }\left(\right.$ film) $/ \mathrm{cm}^{-1}$ $\delta 3360,3258,3082,2923,2861,1671,1606,1473,1452,1387,1252,1217,1052,1024,973,815 .{ }^{1} \mathrm{H}$ NMR $(300 \mathrm{MHz}, \mathrm{DMSO}) \delta_{\mathrm{H}} 8.37(1 \mathrm{H}, \mathrm{d}, \mathrm{J}=9.0 \mathrm{~Hz}, 7-\mathrm{H}), 8.15(\mathrm{~s}, 1 \mathrm{H}, 2-\mathrm{H}), 7.84\left(3 \mathrm{H}, \mathrm{m}, 3^{\prime}-\mathrm{H}\right.$, $\left.22^{\prime \prime}-\mathrm{H}\right), 7.69\left(1 \mathrm{H}, \mathrm{d}, \mathrm{J}=15.3 \mathrm{~Hz}, 2^{\prime}-\mathrm{H}\right), 7.30\left(2 \mathrm{H}, \mathrm{d}, \mathrm{J}=8.2 \mathrm{~Hz}, 32^{\prime \prime}-\mathrm{H}\right), 7.21(1 \mathrm{H}, \mathrm{d}, \mathrm{J}=2.5 \mathrm{~Hz}$, $4-\mathrm{H}), 6.99(1 \mathrm{H}, \mathrm{dd}, \mathrm{J}=9.0, \mathrm{~J}=2.6 \mathrm{~Hz}, 6-\mathrm{H}), 4.86\left(2 \mathrm{H}, \mathrm{d}, \mathrm{J}=2.4 \mathrm{~Hz}, \mathrm{CH}_{2} \mathrm{CCH}\right), 3.58(1 \mathrm{H}, \mathrm{s}$, $\left.\mathrm{J}=2.2 \mathrm{~Hz}, \mathrm{CH}_{2} \mathrm{CCH}\right), 2.91\left(2 \mathrm{H}, \mathrm{t}, \mathrm{J}=6.6 \mathrm{~Hz}, \mathrm{CH}_{2} \mathrm{CH}_{2} \mathrm{NH}\right), 2.75\left(2 \mathrm{H}, \mathrm{J}=6.6 \mathrm{~Hz}, \mathrm{CH}_{2} \mathrm{CH}_{2} \mathrm{NH}\right)$, $2.37(3 \mathrm{H}, \mathrm{s}, \mathrm{O}-\mathrm{CH} 3), 1.94\left(1 \mathrm{H}, \mathrm{sbr}, 1 \mathrm{H}, \mathrm{CH}_{2} \mathrm{CH}_{2} \mathrm{NH}\right) .{ }^{13} \mathrm{C}$ NMR $(75 \mathrm{MHz}, \mathrm{DMSO}) \delta_{\mathrm{C}} 163.9$, $154.3,145.9,141.0,132.2,132.1,130.9,129.8,129.3,124.4,120.2,117.6,117.1,113.8,104.0,79.8$, 78.5, 56.2, 41.7, 29.3, 21.4. HPLC-HRMS (ES ${ }^{+}$) calculated mass for $\mathrm{C}_{23} \mathrm{H}_{22} \mathrm{~N}_{2} \mathrm{O}_{2} 358.1681$; found $\left[(\mathrm{M}+\mathrm{H})^{+}\right] 359.1845 ;\left[(2 \mathrm{M}+\mathrm{H})^{+}\right]$717.3441; purity 96\% (HPLC).

2.1.14. $\mathrm{N}$-(2-(5-Methoxy-1H-indol-3-yl)ethyl)-N-(prop-2-yn-1-yl)prop-2-yn-1-amine (13)

General procedure 2.1.5, 5-methoxytryptamine $(50 \mathrm{mg}, 0.263 \mathrm{mmol})$ in $\mathrm{MeCN}(8 \mathrm{~mL})$, propargyl bromide $(29.3 \mu \mathrm{L}, 0.263 \mathrm{mmol}), 18 \mathrm{~h}$, flash chromatography on silica gel (petroleum ether: EtOAc 0-100\%: MeOH 0-2.5\%) to afford compound 4 as a red oil (57\% yield). $\lambda_{\max }$ (film) $/ \mathrm{cm}^{-1} 3248,3173,2932,2827,1486,1435,1215,1069,790,676,657 .{ }^{1} \mathrm{H}$ NMR $(300 \mathrm{MHz}$, DMSO) $\delta_{\mathrm{H}} 10.59\left(1 \mathrm{H}, \mathrm{s}_{\mathrm{br}}, \mathrm{NH}\right), 7.21(1 \mathrm{H}, \mathrm{d}, \mathrm{J}=8.7 \mathrm{~Hz}, \mathrm{~d}, 7-\mathrm{H}), 7.09(1 \mathrm{H}, \mathrm{d}, \mathrm{J}=2.4 \mathrm{~Hz}, 2-\mathrm{H})$, $7.00(1 \mathrm{H}, \mathrm{d}, \mathrm{J}=2.4 \mathrm{~Hz}, 4-\mathrm{H}), 6.69(1 \mathrm{H}, \mathrm{dd}, \mathrm{J}=8.8, \mathrm{~J}=2.5 \mathrm{~Hz}, 6-\mathrm{H}), 3.75\left(3 \mathrm{H}, \mathrm{s}, \mathrm{O}-\mathrm{CH}_{3}\right), 3.45$ $\left(4 \mathrm{H}, \mathrm{d}, \mathrm{J}=2.3 \mathrm{~Hz}, \mathrm{CH}_{2} \mathrm{CCH}\right), 3.16\left(2 \mathrm{H}, \mathrm{d}, \mathrm{J}=2.3, \mathrm{CH}_{2} \mathrm{CCH}\right), 2.82-2.68\left(4 \mathrm{H}, \mathrm{m}, \mathrm{CH}_{2} \mathrm{CH}_{2} \mathrm{NH}\right.$, $\left.\mathrm{CH}_{2} \mathrm{CH}_{2} \mathrm{NH}\right) ;{ }^{13} \mathrm{C}$ NMR $(75 \mathrm{MHz}, \mathrm{DMSO}) \delta_{\mathrm{C}} 152.9,131.3,127.4,123.2,111.9,111.8,111.0$, 100.0, 79.3, 75.5, 55.3, 52.8, 41.6, 23.0. HPLC-HRMS $\left(\right.$ ES $\left.^{+}\right)$calculated mass for $\mathrm{C}_{17} \mathrm{H}_{18} \mathrm{~N}_{2} \mathrm{O}$ 266.1419; found $\left[(\mathrm{M}+\mathrm{H})^{+}\right]$267.1490; purity 95\% (HPLC).

\subsubsection{3-(2-(Di(prop-2-yn-1-yl)amino)ethyl)-1H-indol-5-yl Cinnamate (14)}

General procedure 2.1.5, 3-(2-aminoethyl)-1H-indol-5-yl cinnamate (107 mg, $0.349 \mathrm{mmol}$ ) in $\mathrm{MeCN}(10 \mathrm{~mL})$, propargyl bromide $(51.9 \mu \mathrm{L}, 0.349 \mathrm{mmol})$ and $\mathrm{Et}_{3} \mathrm{~N}(46.3 \mu \mathrm{L}, 0.349 \mathrm{mmol})$ were added, $72 \mathrm{~h}$, flash chromatography on silica gel (petroleum ether:EtOAc 0-100\%:MeOH $0-3 \%$ ) to afford compound 14 as a yellow oil (39\% yield). $\lambda_{\max }(\mathrm{film}) / \mathrm{cm}^{-1} 3276,2926,2846$, $1709,1628,1449,1240,1203,1171,955,771 .{ }^{1} \mathrm{H}$ NMR $(300 \mathrm{MHz}, \mathrm{DMSO}) \delta_{\mathrm{H}} 10.90\left(1 \mathrm{H}, \mathrm{s}_{\mathrm{br}}, \mathrm{NH}\right)$, 7.89-7.74 (3H, m, 2'-H, 22' $-\mathrm{H}), 7.50-7.42\left(3 \mathrm{H}, \mathrm{m}, 32^{\prime \prime}-\mathrm{H}, 42^{\prime \prime}-\mathrm{H}\right), 7.35(1 \mathrm{H}, \mathrm{d}, \mathrm{H}-7), 7.31(1 \mathrm{H}, \mathrm{d}$, $\mathrm{J}=2.3 \mathrm{~Hz}, 2-\mathrm{H}), 7.23(1 \mathrm{H}, \mathrm{d}, \mathrm{J}=2.3 \mathrm{~Hz}, 4-\mathrm{H}), 6.95-6.82\left(2 \mathrm{H}, \mathrm{m}, 3^{\prime}-\mathrm{H}, 6-\mathrm{H}\right), 3.45(4 \mathrm{H}, \mathrm{d}, \mathrm{J}=2.4$ $\left.\mathrm{Hz}, \mathrm{CH}_{2} \mathrm{CCH}\right), 3.13\left(2 \mathrm{H}, \mathrm{t}, \mathrm{J}=2.2 \mathrm{~Hz}, \mathrm{CH}_{2} \mathrm{CCH}\right), 2.86-2.68\left(4 \mathrm{H}, \mathrm{m}, \mathrm{CH}_{2} \mathrm{CH}_{2} \mathrm{~N}, \mathrm{CH}_{2} \mathrm{CH}_{2} \mathrm{~N}\right)$; ${ }^{13} \mathrm{C}$ NMR $(75 \mathrm{MHz}, \mathrm{DMSO}) \delta_{\mathrm{C}} 168.4,148.4,145.9,136.7,133.4,131.7,131.2,130.0,126.8,120.4$, $117.9,115.1,114.3,113.1,82.0,78.2,55.6,44.2,36.0,25.5$. HPLC-HRMS (ES+) calculated mass for $\mathrm{C}_{25} \mathrm{H}_{22} \mathrm{~N}_{2} \mathrm{O}_{2}$ 382.1681; found [(M+ H) $\left.{ }^{+}\right] 383.1752$; purity 95\% (HPLC).

\subsubsection{3-(2-(Di(prop-2-yn-1-yl)amino)ethyl)-1H-indol-5-yl-(E)-3-( $p$-tolyl) Acrylate (15)}

General procedure 2.1.5, 3-(2-aminoethyl)-1H-indol-5-yl-(E)-3-(p-tolyl) acrylate (95.0 mg, $0.296 \mathrm{mmol})$ in $\mathrm{MeCN}(10 \mathrm{~mL})$, propargyl bromide $(92.9 \mu \mathrm{L}, 0.296 \mathrm{mmol})$ and $\mathrm{Et}_{3} \mathrm{~N}(40 \mu \mathrm{L}$, $0.296 \mathrm{mmol}$ ) were added, $72 \mathrm{~h}$, flash chromatography on silica gel (petroleum ether:EtOAc $0-100 \%: \mathrm{MeOH} 0-2.5 \%)$ to afford compound 15 as a pale-yellow oil (41\% yield). $\lambda_{\max }$ 
(film) $/ \mathrm{cm}^{-1}$ 3309, 3255, 3181, 2926, 2852, 2834, 1725, 1641, 1460, 1312, 1168, 1147, 979, 810. ${ }^{1} \mathrm{H}$ NMR (300 MHz, DMSO) $\delta_{\mathrm{H}} 10.95\left(1 \mathrm{H}, \mathrm{s}_{\mathrm{br}}, \mathrm{NH}\right), 7.86\left(1 \mathrm{H}, \mathrm{d}, \mathrm{J}=16.0 \mathrm{~Hz}, 2^{\prime}-\mathrm{H}\right), 7.74$ $\left(2 \mathrm{H}, \mathrm{d}, \mathrm{J}=8.1 \mathrm{~Hz}, 22^{\prime \prime}-\mathrm{H}\right), 7.39(1 \mathrm{H}, \mathrm{d}, \mathrm{J}=8.7 \mathrm{~Hz}, 7-\mathrm{H}), 7.36-7.29\left(3 \mathrm{H}, \mathrm{m}, 32^{\prime \prime}-\mathrm{H}, 2-\mathrm{H}\right), 7.27$ $(1 \mathrm{H}, \mathrm{d}, \mathrm{J}=2.3 \mathrm{~Hz}, 4-\mathrm{H}), 6.97-6.80\left(2 \mathrm{H}, \mathrm{m}, 3^{\prime}-\mathrm{H}, 6-\mathrm{H}\right), 3.49\left(4 \mathrm{H}, \mathrm{d}, \mathrm{J}=2.4 \mathrm{~Hz}, \mathrm{CH}_{2} \mathrm{CCH}\right), 3.17$ $(2 \mathrm{H}, \mathrm{t}, \mathrm{J}=2.3 \mathrm{~Hz}, \mathrm{CH} 2 \mathrm{CCH}), 2.92-2.72\left(4 \mathrm{H}, \mathrm{m}, \mathrm{CH}_{2} \mathrm{CH}_{2} \mathrm{~N}, \mathrm{CH}_{2} \mathrm{CH}_{2} \mathrm{~N}\right), 2.40\left(3 \mathrm{H}, \mathrm{s}, \mathrm{Ph}_{-} \mathrm{CH}_{3}\right)$; ${ }^{13} \mathrm{C}$ NMR $(75 \mathrm{MHz}, \mathrm{DMSO}) \delta_{\mathrm{C}} 165.8,145.7,143.2,140.7,133.9,131.3,129.6,128.5,127.3$, 116.6, 115.3, 112.4, 111.6, 110.4, 79.2, 75.5, 53.0, 41.5, 22.8, 21.0. HPLC-HRMS (ES ${ }^{+}$) calculated mass for $\mathrm{C}_{26} \mathrm{H}_{24} \mathrm{~N}_{2} \mathrm{O}_{2}$ 396.1838; found $\left[(\mathrm{M}+\mathrm{H})^{+}\right]$397.1909; $\left[(2 \mathrm{M}+\mathrm{H})^{+}\right]$793.3738; purity 95\% (HPLC).

\subsubsection{N-(2-(5-Methoxy-1H-indol-3-yl)ethyl) Propiolamide (16)}

General procedure 2.1.6, DCC (281 mg, $1.36 \mathrm{mmol}), 2$-propynoic acid $(88.1 \mu \mathrm{L}, 1.36 \mathrm{mmol})$ in dry DCM (10 mL), 5-methoxytryptamine (225 mg, $1.18 \mathrm{mmol}), 24 \mathrm{~h}$, flash chromatography on silica gel (petroleum ether: DCM 0-100\%:methanol (MeOH) 0-3\%) to afford compound 7 as a pale red oil (45\% yield). $\lambda_{\max }\left(\right.$ film) $/ \mathrm{cm}^{-1} 3373,3262,2931,2111,1642,1535,1488,1275,1216$, 1032, 802. ${ }^{1} \mathrm{H}$ NMR (300 MHz, DMSO) $\delta_{\mathrm{H}} 10.68(1 \mathrm{H}, \mathrm{sbr}, \mathrm{NH}), 8.84\left(1 \mathrm{H}, \mathrm{s}_{\mathrm{br}}, \mathrm{NHC}(\mathrm{O}) \mathrm{CCH}\right)$, $7.26(1 \mathrm{H}, \mathrm{d}, \mathrm{J}=8.7 \mathrm{~Hz}, \mathrm{~d}, 7-\mathrm{H}), 7.14(1 \mathrm{H}, \mathrm{d}, \mathrm{J}=2.4 \mathrm{~Hz}, 2-\mathrm{H}), 7.05(1 \mathrm{H}, \mathrm{d}, \mathrm{J}=2.5 \mathrm{~Hz}, 4-\mathrm{H}), 6.75$ $(1 \mathrm{H}, \mathrm{dd}, \mathrm{J}=8.7, \mathrm{~J}=2.4 \mathrm{~Hz}, 6-\mathrm{H}), 4.12(1 \mathrm{H}, \mathrm{s}, \mathrm{NHC}(\mathrm{O}) \mathrm{CCH}), 3.80\left(3 \mathrm{H}, \mathrm{s}, \mathrm{O}-\mathrm{CH}_{3}\right), 3.47-3.32(2 \mathrm{H}$, $\left.\mathrm{m}, \mathrm{CH}_{2} \mathrm{CH}_{2} \mathrm{NH}\right), 2.85\left(2 \mathrm{H}, \mathrm{t}, \mathrm{J}=7.4, \mathrm{CH}_{2} \mathrm{CH}_{2} \mathrm{NH}\right) ;{ }^{13} \mathrm{C} \mathrm{NMR}(75 \mathrm{MHz}, \mathrm{DMSO}) \delta_{\mathrm{C}} 153.0,151.6$, 131.3, 127.5, 123.3, 111.9, 111.2, 111.0, 100.1, 78.5, 75.3, 55.3, 39.7, 24.6. HPLC-HRMS (ES+) calculated mass for $\mathrm{C}_{14} \mathrm{H}_{14} \mathrm{~N}_{2} \mathrm{O}_{2}$ 242.1055; found $\left[(\mathrm{M}+\mathrm{H})^{+}\right]$243.1153; [(2M + H $\left.)^{+}\right]$485.2179; $\left[(2 \mathrm{M}+\mathrm{Na})^{+}\right]$507.2001; purity 100\% (HPLC).

\subsubsection{3-(2-Propiolamidoethyl)-1H-indol-5-yl Cinnamate (17)}

General procedure 2.1.6, DCC (58.1 mg, $0.281 \mathrm{mmol}), 2$-propynoic acid $(17.2 \mu \mathrm{L}$, $0.281 \mathrm{mmol})$ in dry DCM $(8 \mathrm{~mL}), 3$-(2-aminoethyl)- $1 H$-indol-5-yl cinnamate $(75.0 \mathrm{mg}$, $0.244 \mathrm{mmol}$ ), $5 \mathrm{~h}$, flash chromatography (petroleum ether:EtOAc 0-100\%) to afford compound 17 as a white solid (89\% yield). $\lambda_{\max }$ (film) / $\mathrm{cm}^{-1} 3386,3299,3224,3054,2936,2107$, $1713,1642,1547,1168,1031,766 .{ }^{1} \mathrm{H}$ NMR (300 MHz, DMSO) $\delta_{\mathrm{H}} 10.94(1 \mathrm{H}, \mathrm{s}$ br, $\mathrm{NH}), 8.80$ $\left(1 \mathrm{H}, \mathrm{s}_{\mathrm{br}}, \mathrm{NHC}(\mathrm{O}) \mathrm{CCH}\right), 7.92-7.75\left(3 \mathrm{H}, \mathrm{m}, 2^{\prime}-\mathrm{H}, 22^{\prime \prime}-\mathrm{H}\right), 7.53-7.41\left(3 \mathrm{H}, \mathrm{m}, 322^{\prime \prime}-\mathrm{H}, 42^{\prime \prime}-\mathrm{H}\right), 7.36$ $(1 \mathrm{H}, \mathrm{d}, \mathrm{J}=8.7 \mathrm{~Hz}, 7-\mathrm{H}), 7.30(1 \mathrm{H}, \mathrm{d}, \mathrm{J}=2.2 \mathrm{~Hz}, 2-\mathrm{H}), 7.22(1 \mathrm{H}, \mathrm{d}, \mathrm{J}=2.4 \mathrm{~Hz}, 4-\mathrm{H}), 6.96-6.82$ $\left(2 \mathrm{H}, \mathrm{m}, 3^{\prime}-\mathrm{H}, 6-\mathrm{H}\right), 4.07(1 \mathrm{H}, \mathrm{s}, \mathrm{NHC}(\mathrm{O}) \mathrm{CCH}), 3.43-3.27\left(2 \mathrm{H}, \mathrm{m}, \mathrm{CH}_{2} \mathrm{CH}_{2} \mathrm{NH}\right), 2.81(2 \mathrm{H}$, $\left.\mathrm{t}, \mathrm{J}=7.4 \mathrm{~Hz}, \mathrm{CH}_{2} \mathrm{CH}_{2} \mathrm{NH}\right) ;{ }^{13} \mathrm{C} \mathrm{NMR}(75 \mathrm{MHz}, \mathrm{DMSO}) \delta_{\mathrm{C}} 165.7,151.6,145.8,143.3,134.0$, $130.7,129.0,128.5,127.2,124.3,117.7,115.4,111.7,110.3,75.4,40.1,24.5$. HPLC-HRMS (ES $\left.{ }^{+}\right)$ calculated mass for $\mathrm{C}_{22} \mathrm{H}_{18} \mathrm{~N}_{2} \mathrm{O}_{3} 358.1317$; found $\left[(\mathrm{M}+\mathrm{H})^{+}\right]$359.1389; purity $100 \%$ (HPLC).

\subsubsection{3-(2-Propiolamidoethyl)-1H-indol-5-yl-(E)-3-(p-tolyl) Acrylate (18)}

General procedure 2.1.6, DCC (55.5 mg, $0.269 \mathrm{mmol}), 2$-propynoic acid $(17.4 \mu \mathrm{L}$, $0.269 \mathrm{mmol})$ in dry DCM $(8 \mathrm{~mL}), 3$-(2-aminoethyl)-1H-indol-5-yl-(E)-3-( $p$-tolyl) acrylate $(75.0 \mathrm{mg}, 0.234 \mathrm{mmol}), 5 \mathrm{~h}$, flash chromatography (petroleum ether: EtOAc 0-100\%) to afford compound 18 as a white solid (78\% yield). $\lambda_{\max }\left(\right.$ film) $/ \mathrm{cm}^{-1} 3270,2925,2106,1717$, $1642,1171,1147,816 .{ }^{1} \mathrm{H}$ NMR $(300 \mathrm{MHz}, \mathrm{DMSO}) \delta_{\mathrm{H}} 10.93\left(1 \mathrm{H}, \mathrm{s}_{\mathrm{br}}, \mathrm{NH}\right), 8.80\left(1 \mathrm{H}, \mathrm{s}_{\mathrm{br}}\right.$, $\mathrm{NHC}(\mathrm{O}) \mathrm{CCH}), 7.81\left(1 \mathrm{H}, \mathrm{d}, \mathrm{J}=16.0 \mathrm{~Hz}, 2^{\prime}-\mathrm{H}\right), 7.69\left(2 \mathrm{H}, \mathrm{d}, \mathrm{J}=7.8 \mathrm{~Hz}, 22^{\prime \prime}-\mathrm{H}\right), 7.35(1 \mathrm{H}$, d, J = 8.5 Hz, d, 7-H), $7.29\left(2 \mathrm{H}, \mathrm{d}, \mathrm{J}=2.3 \mathrm{~Hz}, 3-\mathrm{H}^{\prime \prime}\right), 7.26(1 \mathrm{H}, \mathrm{s}, 2-\mathrm{H}), 7.22(1 \mathrm{H}, \mathrm{m}, \mathrm{H}-4)$, 6.93-6.75 (2H, m, 3'-H, 6-H), 4.07 (1H, s, NHC(O)CCH), 3.44-3.19 (2H, m, $\left.\mathrm{CH}_{2} \mathrm{CH}_{2} \mathrm{NH}\right)$, $2.81\left(2 \mathrm{H}, \mathrm{t}, \mathrm{J}=7.4 \mathrm{~Hz}, \mathrm{CH}_{2} \mathrm{CH}_{2} \mathrm{NH}\right), 2.35\left(3 \mathrm{H}, \mathrm{s}, \mathrm{Ph}-\mathrm{CH}_{3}\right) ;{ }^{13} \mathrm{C} \mathrm{NMR}(75 \mathrm{MHz}, \mathrm{DMSO}) \delta_{\mathrm{C}}$ $165.8,151.5,145.7,143.3,140.7,134.0,131.3,129.6,128.5,127.2,116.5,115.4,111.7,110.3,78.4$, $75.4,39.6,24.5,21.0$. HPLC-HRMS (ES+) calculated mass for $\mathrm{C}_{23} \mathrm{H}_{20} \mathrm{~N}_{2} \mathrm{O}_{3}$ 373.1507; found $\left[(\mathrm{M}+\mathrm{H})^{+}\right]$372.1474; $\left[(2 \mathrm{M}+\mathrm{H})^{+}\right]$745.3009; purity 100\% (HPLC).

\subsection{Biological Evaluation}

2.2.1. AREc32 Cell Line Culture

AREc32 cells, shared by CR Wolf, were grown in Dulbecco's modified Eagle medium (DMEM) with GlutaMAX (Gibco, Invitrogen, Madrid, Spain), supplemented with 10\% 
$(v / v)$ filtrated fetal bovine serum (FBS; Gibco, Invitrogen, Madrid, Spain), $1 \%$ of antibiotics penicillin-streptomycin, and geneticin ( $0.8 \mathrm{mg} / \mathrm{mL}$ G418; Gibco, Invitrogen, Madrid, Spain). Cells were maintained at $37^{\circ} \mathrm{C}$ under humidified atmosphere $\left(5 \% \mathrm{CO}_{2}\right.$ and $95 \%$ relative humidity). Cells were cultured in flasks (Corning, Madrid, Spain) until reaching 80\% confluence and sub-cultured using 0.25\% EDTA-trypsin (Thermo Fisher, Madrid, Spain) for $5 \mathrm{~min}$. They were recovered by centrifugation at $800 \mathrm{rpm}$ for $10 \mathrm{~min}$. Cells were used from the third to twelfth passage.

\subsubsection{Luciferase Activity: NRF2 Induction}

AREc32 cells were stably transfected with the plasmid pGL-8xARE that implements 8 copies of the antioxidant response element (ARE) sequences followed by luciferase reporter gen. Therefore, NRF2 induction can be related to the activation of ARE sequences by measuring luciferase activity in terms of luminescence production. For NRF2 induction experiments, cells were plated in 96 white flat bottom well plates (20,000 cells/well). After $24 \mathrm{~h}$, cells were incubated with selected compounds at the desired concentrations in duplicates for $24 \mathrm{~h}$. The Luciferase Assay System (Promega E1500) was used according to provider protocol and luminescence was quantified in an Orion II microplate luminometer (Berthold, Germany). Fold induction of luciferase activity was normalized to basal conditions. Data were expressed as CD values, which means the concentration needed to duplicate the luciferase activity compared to basal conditions. CD values were calculated from dose-response curves fitted by non-linear regression for each compound after logarithmic transformation of the data using GraphPad Prism 8.0 software.

\subsubsection{Inhibition of Monoamine Oxidase Enzymes (MAO-A and MAO-B)}

Assays were performed in 96 black flat bottom well plates in a final volume of $200 \mu \mathrm{L} /$ well. Test compounds at the desired concentrations were pre-incubated with human recombinant monoamine oxidase enzymes hrMAO-A or hrMAO-B (Sigma-Aldrich, Madrid, Spain) at final concentrations of 0.0180 or $0.0135 \mathrm{U} / \mathrm{mL}$, respectively, during $30 \mathrm{~min}$ at $37^{\circ} \mathrm{C}$. Reaction was started by adding Amplex UltraRed reagent $(12.5 \mu \mathrm{M}$ final concentration; Invitrogen, Madrid, Spain), horseradish peroxidase $(0.02 \mathrm{U} / \mathrm{mL}$ final concentration; Sigma-Aldrich, Spain), and tyramine ( $0.5 \mathrm{mM}$ final concentration; SigmaAldrich, Spain). Resorufin production was measured at 530/590 nm (excitation/emission) during $30 \mathrm{~min}$ in a FluoStar Optima (BMG Labtech, Ortenberg, Germany) fluorescence plate reader. All solutions were prepared in $25 \mathrm{mM}$ of sodium phosphate buffer $\mathrm{pH} 7.4$. Clorgyline (MAO-A selective inhibitor) and rasagiline (MAO-B selective inhibitor) were used as positive controls of inhibition. $\mathrm{IC}_{50}$ values for MAO-A and MAO-B inhibition were calculated from dose-response curves obtained by non-linear regression fitting after logarithmic transformation of the data using GraphPad Prism 8.0 software.

\subsubsection{MAO-A and MAO-B Reversibility Assays}

Assays were performed following the general procedure 2.15: Inhibition of monoamine oxidase enzymes (MAO-A and MAO-B), modifying in this case the pre-incubation time. Briefly, 0-, 15-, and 30-min pre-incubation times were used to obtain MAO inhibition activities versus time curves. MAO inhibition activity varies over time for irreversible inhibitors; however, it remains stable for reversible type inhibitors. Rasagiline and clorgyline were used as references of irreversible inhibition for comparative purposes and each compound was incubated at a $1.8 \times \mathrm{IC}_{50}-\mu \mathrm{M}$ final concentration to observe adequate $\mathrm{MAO}$ inhibition.

\subsubsection{Oxygen Radical Absorbance Capacity (ORAC) Assay}

The ORAC test [59] was performed to evaluate the oxygen free radical scavenger capacity of the compounds. ( \pm )-6-hydroxy-2,5,7,8-tetramethylchromane-2-carboxylic acid (Trolox) at 1, 2, 4, 6, and $8 \mu \mathrm{M}$ concentrations was used as reference compound and melatonin was used as positive control. Compounds at desired concentrations $(0.03,0.1$, $0.3,1,3$, and $10 \mu \mathrm{M}$ ) were diluted in phosphate-buffered saline (PBS; $10 \mathrm{mM}, \mathrm{pH} 7.4$ ) 
at $37{ }^{\circ} \mathrm{C}$ and placed in a 96 black flat bottom well plate. Then $150 \mu \mathrm{L}$ of fluorescein (70 nM final concentration), $25 \mu \mathrm{L}$ of PBS buffer for blank, $25 \mu \mathrm{L}$ of Trolox solution for standard, and $25 \mu \mathrm{L}$ of melatonin or compound were added to each well. A fluorescence measurement was done first to determine the basal signal. Then, $25 \mu \mathrm{L}$ of 2 , 2' ${ }^{\prime}$-azobisamidinopropane dihydrochloride (AAPH) (12 mM final concentration) was quickly added. All samples were measured in duplicates in three different experiments. Fluorescence was recorded at $485 / 520 \mathrm{~nm}$ (excitation/emission) for $90 \mathrm{~min}$ at $37^{\circ} \mathrm{C}$ to obtain the area under the fluorescence decay curve (AUC) in a FLUOstar Optima plate reader (BMG Labtech, Ortenberg, Germany). Results were expressed in terms of Trolox equivalents (T.eq.), obtained by plotting net AUC versus compound concentration, then performing linear regression and normalizing slopes of the compounds considering Trolox as reference.

\subsubsection{Blood-Brain Barrier Permeation Assay (PAMPA)}

Prediction of the compounds' ability to cross the blood-brain barrier (BBB) by passive diffusion was evaluated using the parallel artificial membrane permeation assay (PAMPA), as described previously [60]. Briefly, permeability of the compounds was measured at an initial concentration of $100 \mu \mathrm{M}$, including positive and negative controls (Supplementary Data; Table S3). To model the BBB, the filter membrane of the 96-well donor plate (multiscreen IP sterile clear plate PDVF membrane, pore size $0.45 \mu \mathrm{M}$ ) was filled with $4 \mu \mathrm{L}$ of porcine brain lipid (PBL, Avanti Polar Lipids, Inc., Birmingham, UK) dissolved in dodecane $(20 \mathrm{mg} / \mathrm{mL}$, Sigma-Aldrich, Madrid, Spain). After $5 \mathrm{~min}, 180 \mu \mathrm{L}$ of each compound solution in PBS (10 mM, pH 7.4) was added to determine their ability to pass the membrane $\left(V_{D}\right)$. The 96-well acceptor plate (Multiscreen, Millipore Corp., Madrid Spain) was loaded with $180 \mu \mathrm{L}$ of PBS $\left(\mathrm{V}_{\mathrm{A}}\right)$. Then, the donor filter plate was carefully placed on the acceptor plate to form a sandwich-like system, which was left undisturbed for $4 \mathrm{~h}$ at room temperature. After incubation, the donor plate was removed and the absorbance at the maximum absorption wavelength for each compound was recorded in both the acceptor and donor wells $(150 \mu \mathrm{L} /$ well) using a UV plate reader SPECTROstar Nano (BMG Labtech, Ortenberg, Germany). Concentration of the compounds in the donor and acceptor plates and equilibrium concentration were calculated and expressed as permeability (Pe) according the Equation (1). Results are expressed as mean \pm standard error of the mean (SEM) of at least three different experiments in duplicate:

$$
\log \mathrm{Pe}=\log \left\{\mathrm{C} \times-\ln \left(1-\frac{[\mathrm{drug}]_{\text {acceptor }}}{[\mathrm{drug}]_{\text {equilibrium }}}\right)\right\}
$$

where $C=\left(\frac{\mathrm{V}_{\mathrm{D}} \times \mathrm{V}_{\mathrm{A}}}{\left(\mathrm{V}_{\mathrm{D}}+\mathrm{V}_{\mathrm{A}}\right) \times \text { Area } \times \text { Time }}\right)$.

\subsubsection{SH-SY5Y Neuroblastoma Cell Line Culture}

SH-SY5Y human neuroblastoma cell line (ECACC, 94030304) was grown in a modified minimum essential medium (MEM) (4.765 g/L MEM; $2.5 \%$ minimum essential mediumnon-essential amino acids (Invitrogen, Madrid, Spain); Ham's F12 Nutrients Mix (Thermo Fisher, Massachusetts, EEUU); $0.5 \mathrm{mM}$ of sodium pyruvate (Sigma-Aldrich, Madrid, Spain); $2 \mathrm{~g} / \mathrm{L}$ of $\mathrm{NaHCO}_{3}$ (PanReac, Barcelona, Spain); 10\% (v/v) filtrated FBS (Gibco, Invitrogen, Madrid, Spain), and $100 \mathrm{U} / \mathrm{mL}$ of penicillin/streptomycin (Invitrogen, Spain)). Cells were maintained at $37{ }^{\circ} \mathrm{C}$ under humidified atmosphere $\left(5 \% \mathrm{CO}_{2}\right.$ and $95 \%$ relative humidity). Cells were cultured in flasks (Corning, Madrid, Spain) until reaching $80 \%$ confluence and sub-cultured using 0.25\% EDTA-trypsin (Thermo Fisher, Madrid, Spain) for 5 min. They were recovered by centrifugation at $800 \mathrm{rpm}$ for $10 \mathrm{~min}$. Cells from the third to twelfth passage were seeded at a density of 80,000 cells/well in 96-well plates for the neuroprotection studies. 


\subsubsection{Neuroprotection Studies in the SH-SY5Y Neuroblastoma Cell Line}

Cells were seeded at a density of 60,000 cells/well in 96-well transparent plates for $24 \mathrm{~h}$. Then cells were pre-incubated with the corresponding compound at $0.1 \mu \mathrm{M}$. After $24 \mathrm{~h}$, the medium was removed and replaced with 1\% $(v / v)$ FBS media containing the corresponding compound at $0.1 \mu \mathrm{M}$ and the selected toxic stimuli, namely a mixture of rotenone and oligomycin A (R/O; 30/10 $\mu \mathrm{M}$, respectively; Sigma-Aldrich, Spain) or 6hydroxydopamine (100 $\mu \mathrm{M}$; Sigma-Aldrich, Spain). The co-incubation of toxic stimuli plus compounds was maintained for $24 \mathrm{~h}$. Melatonin and rasagiline were used as references for comparative purposes. After the co-incubation period, cell viability was assessed by the 3-(4,5-dimethylthiazol-2-yl)-2,5-diphenyltetrazolium bromide (MTT) method considering basal conditions as $100 \%$ survival.

\subsubsection{Ethics for Animal Experimentation}

All experimental procedures were performed following the Guide for the Care and Use of Laboratory Animals, in accordance with the European Guidelines for the use and care of animals for research in accordance with the European Union Directive of 22 September 2010 (2010/63/UE) and with the Spanish Royal Decree of 1 February 2013 (53/2013).

\subsubsection{Mixed Primary Glial Culture}

Mixed primary glial cultures were obtained from cerebral cortex of 2-5-day-old Sprague-Dawley rats. Blood vessels and meninges were first removed in Hank's balanced salt solution (HBSS) and then forebrains were dissociated in DMEM/F12 medium. After mechanical dissociation, filtering, and centrifugation at $800 \mathrm{rpm}$ for $10 \mathrm{~min}$, cells were plated at a density of 30,000 cells/well in DMEM/F12 medium with 20\% $(v / v)$ FBS and $1 \%$ penicillin/streptomycin (10,000 units). Cells were maintained at $37{ }^{\circ} \mathrm{C}$ under humidified atmosphere ( $5 \% \mathrm{CO}_{2}$ and $95 \%$ relative humidity). After 5 days, medium was substituted by DMEM/F12 medium with 10\% $(v / v)$ FBS. Cells were cultured for 7-10 days before treatment.

\subsubsection{Nitrite Production Measurement in Mixed Primary Glial Cultures}

Primary glial cells were pre-treated with compounds at the desired concentration for $24 \mathrm{~h}$. Then, treatments were removed and glial cells were incubated with lipopolysaccharide (LPS, $1 \mu \mathrm{g} / \mathrm{mL}$; Sigma-Aldrich, Spain) in the presence of each compound at the desired concentration for $18 \mathrm{~h}$. Nitrite production was then assessed by modified Griess assay [61]. Briefly, $150 \mu \mathrm{L}$ of each sample was mixed with $75 \mu \mathrm{L}$ of 4,4'-diamino-diphenylsulfone (Dapsone) and $75 \mu \mathrm{L}$ of $\mathrm{N}$-(1-naphthyl)ethylenediamine (Neda). The mixture was incubated at room temperature for $5 \mathrm{~min}$. Then, absorbance was measured at $550 \mathrm{~nm}$ in a microplate reader SPECTROstar Nano (BMG Labtech, Ortenberg, Germany). Data were normalized to basal conditions, setting this value as $100 \%$ of nitrite production. $\mathrm{EC}_{50}$ values were calculated from dose-response curves obtained from representing percentage of nitrites reduction vs. compound concentration.

\subsubsection{Quantification of IL-1 $\beta$ Levels by ELISA}

Supernatants obtained from mixed primary glial cell cultures were used to determine IL-1 $\beta$ levels by quantitative ELISA kits (Preprotech, R\&D Systems-bioNova, Madrid, Spain) following the manufacturer's instructions. Commercial solution of IL-1 $\beta$ was used to generate a standard curve for sample quantification. Absorbance was measured in a microplate reader SPECTROstar Nano (BMG Labtech) at 405 with $650 \mathrm{~nm}$ correction.

\subsubsection{Acute Treatment of Rat Striatal Slices}

Three- to four-month-old Sprague-Dawley rats were decapitated, the brain was removed, and the two brain hemispheres were separated. Each hemisphere was then cut into 300- $\mu \mathrm{m}$ coronal slices using a Mcllwain Tissue Chopper (Cavey Laboratory Engineering, Surrey, UK) and separated in a previously oxygenated $\left(95 \% \mathrm{O}_{2}\right.$ and $\left.5 \% \mathrm{CO}_{2}\right)$ ice-cold 
Krebs's dissection buffer $\left(120 \mathrm{mM}\right.$ of $\mathrm{NaCl} ; 2 \mathrm{mM}$ of $\mathrm{KCl} ; 26 \mathrm{mM}$ of $\mathrm{NaHCO}_{3} ; 1.18 \mathrm{mM}$ of $\mathrm{KH}_{2} \mathrm{PO}_{4} ; 10 \mathrm{mM}$ of $\mathrm{MgSO}_{4} ; 0.5 \mathrm{mM}$ of $\mathrm{CaCl}_{2} ; 11 \mathrm{mM}$ of glucose; and $200 \mathrm{mM}$ sucrose at pH 7.4) using a Leica SE6 microscope (Leica, Madrid, Spain). Striatum-containing slices were selected and immediately incubated for stabilization in dissection buffer without sucrose, bubbled with $95 \% \mathrm{O}_{2}$ and $5 \% \mathrm{CO}_{2}$ for $45 \mathrm{~min}$ at $34{ }^{\circ} \mathrm{C}$, allowing slices to recover from slicing injury. Afterwards, brain slices were placed in a 24-well plate containing new medium composed of 1:1 control buffer $\left(120 \mathrm{mM}\right.$ of $\mathrm{NaCl} ; 2 \mathrm{mM}$ of $\mathrm{KCl} ; 26 \mathrm{mM}$ of $\mathrm{NaHCO}_{3}$; $1.18 \mathrm{mM}$ of $\mathrm{KH}_{2} \mathrm{PO}_{4} ; 1.19 \mathrm{mM}$ of $\mathrm{MgSO}_{4} ; 2 \mathrm{mM}$ of $\mathrm{CaCl}_{2}$; and $11 \mathrm{mM}$ of glucose) and DMEM/F12 medium (Invitrogen, Madrid, Spain). Slices were exposed for $4 \mathrm{~h}$ to 6-OHDA $(100 \mu \mathrm{M})$ or rotenone $(10 \mu \mathrm{M})$, and co-incubated with corresponding compounds $(10 \mu \mathrm{M})$. Saline was used instead of toxics for basal conditions. Finally, cell death and the ROS production were measured.

\subsubsection{Measurement of Cell Death and ROS Production in Rat Striatal Slices}

ROS quantification was performed using the fluorescent probe 2,7'-dichlorodihydrofluorescein diacetate $\left(\mathrm{H}_{2} \mathrm{DCFDA}, \lambda_{\mathrm{Ex} / \mathrm{Em}}=495 / 529 \mathrm{~nm}\right.$; Thermo Fisher, Madrid, Spain). Cell death quantification was performed using the fluorescent probe propidium iodide (PI, $\lambda_{\mathrm{Ex} / \mathrm{Em}}=535 / 617$ $\mathrm{nm}$; Thermo Fisher, Madrid, Spain). Brain slices were incubated with both probes for $45 \mathrm{~min}$ at a concentration of $10 \mu \mathrm{L} / \mathrm{mL}$ for $\mathrm{H}_{2}$ DCFDA and $1 \mu \mathrm{L} / \mathrm{mL}$ for PI, in the presence of $1 \mu \mathrm{L} / \mathrm{mL}$ of Hoechst at $37^{\circ} \mathrm{C}$. Fluorescence from striatum slices was then recorded for $\mathrm{H}_{2} \mathrm{DCFDA}\left(\lambda_{\mathrm{Ex} / \mathrm{Em}}\right.$ $=495 / 529 \mathrm{~nm}), \mathrm{PI}\left(\lambda_{\mathrm{Ex} / \mathrm{Em}}=535 / 617 \mathrm{~nm}\right)$, and Hoechst $\left(\lambda_{\mathrm{Ex} / \mathrm{Em}}=350 / 461 \mathrm{~nm}\right)$, in an inverted Nikon Eclipse T2000-U microscope (Nikon, Tokyo, Japan). NIS-Elements BR 4.10.04 64-bit software was used to analyze the images.

\subsubsection{MEF Cell Lines Culture}

Mouse embryonic fibroblasts (MEFs) obtained from NRF2 ${ }^{+/+}$(wild-type; WT) and $\mathrm{NRF}^{-/-}$(knock-out; KO) mice [62] were grown in DMEM with GlutaMAX (Gibco, Invitrogen, Spain), supplemented with $10 \%(\mathrm{v} / \mathrm{v})$ filtrated fetal bovine serum (FBS; Gibco, Invitrogen, Madrid, Spain), and 1\% of antibiotics penicillin-streptomycin. Cells were maintained at $37{ }^{\circ} \mathrm{C}$ under humidified atmosphere $\left(5 \% \mathrm{CO}_{2}\right.$ and $95 \%$ relative humidity). Cells were cultured in flasks (Corning, EEUU) until reaching $80 \%$ confluence and sub-cultured using $0.25 \%$ EDTA-trypsin (Thermo Fisher, EEUU) for $5 \mathrm{~min}$. They were recovered by centrifugation at $800 \mathrm{rpm}$ for $10 \mathrm{~min}$. Cells from the third to twelfth passage were seeded at a density of 500,000 cells/well in 6-well plates for western blot experiments.

\subsubsection{Western Blot}

Cell samples were lysed in ice-cold AKT lysis buffer $(137 \mathrm{mM}$ of $\mathrm{NaCl}, 20 \mathrm{mM}$ of $\mathrm{NaF}, 10 \%$ glycerol, $20 \mathrm{mM}$ of Tris- $\mathrm{HCl}, 1 \%$ Nonidet $\mathrm{P}-40,1 \mu \mathrm{g} / \mathrm{mL}$ of leupeptin, $1 \mathrm{mM}$ of phenylmethylsulfonylfluoride, $1 \mathrm{mM}$ of sodium pyrophosphate, and $1 \mathrm{mM}$ of $\mathrm{Na}_{3} \mathrm{VO}_{4}, \mathrm{pH}$ 7.5). Protein quantification was performed using the Pierce BCA Protein Assay Kit (ThermoFisher, Madrid, Spain) following manufacturer's instructions. Then, $30 \mu \mathrm{g}$ of protein were resolved by sodium dodecyl sulfate-polyacrylamide gel electrophoresis (SDS-PAGE) and transferred to Immobilon-P polyvinylidene difluoride (PVDF) membranes (Millipore Corp.). Membranes were activated with methanol and blocked with $4 \%$ bovine serum albumin (BSA) in Tris-buffered saline-Tween (TTBS: $10 \mathrm{mM}$ of Tris, $150 \mathrm{mM}$ of $\mathrm{NaCl} ; 0.2 \%$ Tween-20, pH 7.4) for $2 \mathrm{~h}$. After that, membranes were incubated overnight at $4{ }^{\circ} \mathrm{C}$ with the corresponding primary antibodies: anti-HMOX1 (1:1000, ab68477, Abcam, Cambridge, UK), anti-glutamate-cysteine ligase catalytic subunit (GCLC, 1:1000, ab41463, Abcam), anti$\beta$-actin (1:50,000, A3854, Sigma-Aldrich, Madrid, Spain). Peroxidase-conjugated secondary antibodies (1:10,000, Santa-Cruz Biotechnology, Heidelberg, Germany) were used after TTBS washings for $1 \mathrm{~h}$ to detect proteins by enhanced chemiluminescence using the ECL Advance Western-blotting Detection Kit (GE Healthcare, Amersham, The Netherland) and the ChemiDoc MP System (Bio-Rad Laboratories, Madrid, Spain). Band intensities from specific proteins were analyzed with Fiji software. 


\subsubsection{MTT Method for Cell Viability Measurement}

After treatments, primary mixed glial cultures and SH-SY5Y, AREc32, and MEF cells were incubated for 120 min with tetrazolium salt (3-(4,5-dimethylthiazol-2-yl)-2,5diphenyltetrazolium bromide solution (MTT, $0.5 \mathrm{mg} / \mathrm{mL}$ ) for cell viability measurement. In this assay, MTT (yellow salt) was reduced to purple insoluble formazan crystals by oxidoreductase enzymes from viable cells. Then, the formazan crystals were solubilized by adding dimethyl sulfoxide (DMSO) to the corresponding p96-well plates. Finally, absorbance was measured at $535 \mathrm{~nm}$ in a microplate reader SPECTROstar Nano (BMG Labtech, Ortenberg, Germany). Basal absorbance was set to $100 \%$ and results were normalized to basal conditions.

\subsubsection{Molecular Docking on MAO-B}

Docking was performed with Schrödinger software using Glide [63]. First, ligand states were produced at pH 7.4 using Epik [64]. Afterwards, they were prepared and minimized using Lig-Prep module [65]. PDB-ID structures were prepared and minimized with the Protein Preparation Wizard tool in Maestro using Optimized Potentials for Liquid Simulations 3 (OPLS3) force field [66]. The box for docking calculations was placed to cover the bipartite MAO-B cavity (both entrance and substrate cavities). Best poses were inspected visually and ranked by energy. The glide ligand docking algorithm was used with standard precision as docking method. All images were constructed with PyMOL software [67].

\subsubsection{Molecular Dynamics (MD) Simulations}

The selected complexes from docking were employed for MD simulations for $300 \mathrm{~ns}$ using Amber18 suite [68]. Ligand parameters were obtained using the Antechamber package in Amber18. In brief, partial charges were attributed with the AM1-BCC charge method [69], employing the general AMBER force field (GAFF) atom types [70]. Ff14SB force field was used for protein parameters and the TIP3P water model was used for proteinligand complex solvation. Sodium and chloride ions were included to mimic physiological concentration of $0.15 \mathrm{M}$. Afterwards, the system was submitted to 500 steps of the steepest descent algorithm followed by 500 steps of the conjugate gradient algorithm. An initial $100 \mathrm{kcal} \mathrm{mol}^{-1} \cdot \mathrm{A}^{-2}$ harmonic potential restriction was applied to the complex and it was gradually lowered. Then, the whole system was minimized and it was heated from 0 to $310 \mathrm{~K}$ using the Langevin thermostat in the canonical ensemble (NVT) with complex 2 harmonic potential restriction of $2 \mathrm{kcal} \mathrm{mol}^{-1} \cdot \mathrm{A}^{-2}$. Before starting the production run, the system was finally equilibrated at $310 \mathrm{~K}$ in the isothermal-isobaric ensemble (NPT) without harmonic limitation. Analysis of the trajectories was performed using the cpptraj module [71] of Amber18 and they were visualized using VMD [72]. Images of MD simulations were constructed using Pymol software [67].

\subsubsection{Statistical Analysis}

Data are represented as mean \pm S.E.M. $\mathrm{IC}_{50}$ and $\mathrm{LD}_{50}$ values were calculated by nonlinear regression analysis of individual dose-response curves. Experimental and control groups were compared using the $t$-test and multiple groups were compared using a oneway analysis of variance test (one-way ANOVA) followed by a Newman-Keuls post hoc test. Statistical significance was set at ${ }^{*} p<0.033,{ }^{* *} p<0.002$, and ${ }^{* * *} p<0.001$. Data was analyzed using GraphPad Prism 8.0 software.

\section{Results and Discussion}

\subsection{Synthesis of the 2-(1H-Indol-3-yl)ethan-1-amine Derivatives}

Synthesis of the 2-(1H-indol-3-yl)ethan-1-amine derivatives 10-18 was performed starting from 5-methoxytryptamine, 3-(2-aminoethyl)-1H-indol-5-yl-(E)-3-( $p$-tolyl) acrylate or 3-(2-aminoethyl)- $1 H$-indol-5-yl cinnamate as shown in Scheme 1. Amine derivatives 10 and 13 were obtained from 5-methoxytryptamine in one reaction by nucleophilic addition 
to propargyl bromide in presence of $\mathrm{Et}_{3} \mathrm{~N}$. Derivative $\mathbf{1 6}$ is readily available by amidation reaction with propiolic acid using DCC as coupling reagent. Amine intermediates $\mathbf{8}$ and $\mathbf{9}$ were prepared from the hydroxy-derivative 2 by Boc protection of amine group followed by Steglich type esterification with the corresponding acrylate 4 or 5 in the presence of DCC as a coupling reagent and HOBt as catalyst. Amine intermediates 8-9 were finally obtained in high yields by deprotection of the Boc group from 6-7 in presence of TFA. Final compounds $\mathbf{1 1}, \mathbf{1 2}, \mathbf{1 4}$, and 15 were then synthesized with moderate yields in one-step reaction from 8-9 by nucleophilic addition to propargyl bromide in presence of $\mathrm{Et}_{3} \mathrm{~N}$. Amide derivatives 17 and 18 were obtained from 8-9 by catalytic amidation using the same conditions previously described for 16. Physicochemical properties predictions for compounds 10-18 are shown in Table S1.

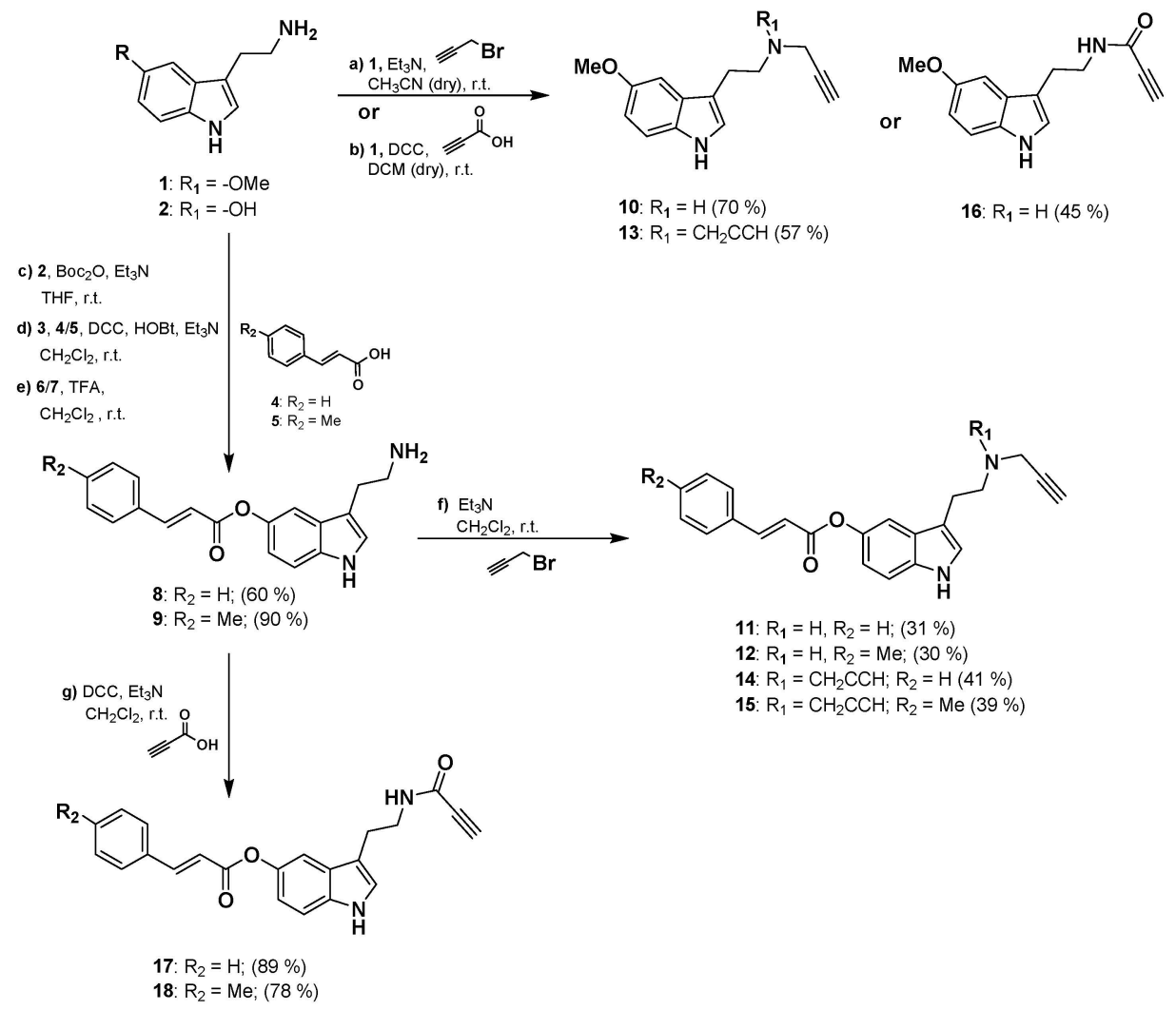

Scheme 1. General procedure for the synthesis of 10-18 compounds.

\subsection{Pharmacological Evaluation}

\subsubsection{NRF2 Induction and MAO Inhibition}

As introduced, compounds 10-18 were designed as NRF2 inducers. They bear an aryl-acrylate moiety designed to react with key KEAP1 cysteine residues, as previously described by our group [73]. In addition, the different acetylene substitution pattern is expected to modulate this activity based on its electrophilic character. Thus, we first investigated the NRF2 induction potential of our novel derivatives using the stable human mammary MCF7-derived reporter cell line AREc32 [74]. We performed dose-response curves to calculate the corresponding CD values (concentration required to double luciferase expression), summarized in Table 1. In general, novel compounds showed moderate NRF2 induction activity with CD values at the low micromolar range, arising interesting structure activity relationships. As expected, aryl-acrylate moiety inclusion increased NRF2 induction capacity compared to the corresponding -OMe derivatives (10, 13, and 16). Compounds 11 and $12(C D=1.80 \pm 0.28$ and $2.99 \pm 0.26 \mu \mathrm{M}$, respectively) showed 7- and 10 -fold potentiation compared to $10(C D=20.9 \pm 2.20)$; compounds 14 and $15(C D=5.07$ \pm 0.23 and $5.76 \pm 0.07 \mu \mathrm{M}$, respectively) showed a 3-fold potentiation compared to 13 
$(C D=13.9 \pm 1.5 \mu \mathrm{M})$. These results are in line with the increased electrophilic character of included aryl-acrylate moieties related to their Cys modification mechanism of action to induce NRF2. Considering amine derivatives 10-15, compounds with a single acetylene moiety (i.e., 10, 11, and 12) showed, in general, a slight increase in NRF2 induction activity, possibly due to steric hindrances of double substituted derivatives at regulatory Cys-residues binding sites. Regarding substituents at aryl-acrylate aromatic ring, phenyl derivatives exhibited increased potency compared $p$-tolyl $(\mathrm{CD}=1.80 \pm 0.28$ and $5.07 \pm$ $0.23 \mu \mathrm{M}$ for compounds 11 and 14, respectively, compared to $\mathrm{CD}=2.99 \pm 0.26$ and 5.76 $\pm 0.075 \mu \mathrm{M}$ for compounds 12 and 15). These results could be explained by the positive inductive effect of methyl group leading to a reduced electrophilic character. Considering amide derivatives 16,17 , and 18, the propynyl substituent is conjugated with an amide electronic system implementing strong electrophiles resulting in higher potency (e.g., $\mathrm{CD}$ $=1.35 \pm 0.12 \mu \mathrm{M}$ for compound 16). This hypothesis is in line with cellular toxicity observed for these compounds, as potent electrophiles could lead to a glutathione depletion and unwanted toxicity [75] (Supplementary Data; Table S2). Proof thereof is the fact that compounds 17 and 18, including aryl-acrylate moieties compared to 16, showed no activity in this assay due to their high toxicity. Importantly, novel compounds demonstrated an improved profile compared to reference compounds melatonin and rasagiline.

Table 1. Main targets validation for compounds 10-18. NRF2 induction activity in the AREc32 cell line model and MAO inhibition of compounds 10-18 and reference compounds. NRF2 induction CD values represent the concentration of compound required to double luciferase activity compared to basal conditions. Tert-Butylhydroquinone (TBHQ) was used as positive control of NRF2 induction. $\mathrm{MAO}$ inhibition $\mathrm{IC}_{50}$ values were calculated from dose-response curves. Data are expressed as mean \pm SEM of at least 3 independent experiments.

\begin{tabular}{|c|c|c|c|c|}
\hline Compound & $\begin{array}{c}\text { CD }(\mu \mathrm{M}) \\
\text { NRF2 Induction }\end{array}$ & $\begin{array}{c}\mathrm{IC}_{50} \mathrm{MAO}-\mathrm{B} \\
(\mu \mathrm{M})\end{array}$ & $\begin{array}{c}\mathrm{IC}_{50} \text { MAO-A } \\
(\mu \mathrm{M})\end{array}$ & $\begin{array}{l}\text { Selectivity Index } \\
\text { (MAO-A/MAO-B) }\end{array}$ \\
\hline Melatonin & $>30$ & $>100$ & $>100$ & - \\
\hline Rasagiline & NA & $0.010 \pm 0.0011$ & $>0.1$ & $>10$ \\
\hline TBHQ & $1.82 \pm 0.09$ & NE & NE & NE \\
\hline 10 & $20.9 \pm 2.20$ & $77.6 \pm 9.10$ & $0.826 \pm 0.11$ & 0.0106 \\
\hline 11 & $1.80 \pm 0.28$ & $3.00 \pm 0.21$ & $43.8 \pm 6.5$ & 14.6 \\
\hline 12 & $2.99 \pm 0.26$ & $3.03 \pm 0.27$ & $59.1 \pm 6.0$ & 19.5 \\
\hline 13 & $13.9 \pm 1.50$ & $89.1 \pm 4.30$ & $>100$ & $>1.12$ \\
\hline 14 & $5.07 \pm 0.23$ & $17.0 \pm 1.60$ & $56.6 \pm 6.3$ & 3.33 \\
\hline 15 & $5.76 \pm 0.07$ & $28.1 \pm 0.60$ & $>100$ & $>3.56$ \\
\hline 16 & $1.35 \pm 0.12$ & $>100$ & $>100$ & - \\
\hline 17 & NA & $50.0 \pm 2.90$ & $>100$ & $>2.00$ \\
\hline 18 & NA & $13.9 \pm 1.20$ & $81.9 \pm 5.4$ & 5.89 \\
\hline
\end{tabular}

NA: not active; NE: not evaluated.

Considering MAO-B implication in PD physiopathology and its clinical significance [6], we designed our novel compounds to selectively inhibit this isozyme. Aryl-acrylate moiety inclusion to melatonin scaffold led to novel structures sharing important pharmacophoric features with known inhibitors [76]. Acetylene moieties, which are commonly present in irreversible MAO-B inhibitors, could increase and modulate compounds potency. Therefore, we performed MAO enzymatic assays with dose-response curves to determine the inhibitory capacity and selectivity of compounds $\mathbf{1 0 - 1 8}$. As shown in Table 1, compounds showed, in general, moderate MAO-B inhibition with $\mathrm{IC}_{50}$ values at the micromolar range. Remarkably, all compounds were MAO-B selective inhibitors, except derivative 10 that showed MAO-A selectivity, and compound $\mathbf{1 6}$ that was not active. Structure-activity evaluation demonstrates that aryl-acrylate moiety presence is related to higher inhibitory activities (compounds 11 and 12, $\mathrm{IC}_{50}=3.00 \pm 0.21$ and $3.03 \pm 0.27 \mu \mathrm{M}$, respectively, compared to $10, \mathrm{IC}_{50}=77.6 \pm 9.1 \mu \mathrm{M}$; compounds 14 and $15, \mathrm{IC}_{50}=17.0 \pm 1.6$ and $28.1 \pm$ $0.60 \mu \mathrm{M}$, respectively, compared to $13, \mathrm{IC}_{50}=89.1 \pm 4.3 \mu \mathrm{M}$; compounds 17 and $18, \mathrm{IC}_{50}=$ 
$50.0 \pm 2.9$ and $13.9 \pm 1.2 \mu \mathrm{M}$, respectively compared to $16, \mathrm{IC}_{50}>100 \mu \mathrm{M}$ ). This moiety is also related with increased selectivity, these derivatives being at least 2-fold more potent towards MAO-B isozyme compared to MAO-A (compounds 11, 12, 14, 15, 16, and 17). These results might be explained by additional aromatic interactions stablished between this motif and key residues at MAO-B active site.

\subsubsection{Antioxidant Activity, Anti-Inflammatory Properties, and Blood-Brain Barrier} Permeation Capacity

As introduced, OS plays a central role in PD onset and progression [1,5]. Considering the structural relationship between novel compounds and the free radical scavenger melatonin, it is expected that derivatives 10-18 exert a considerable antioxidant activity since melatonin has a direct free radical scavenging effect [77]. Therefore, we used the ORAC assay to test their oxygen derived free radical scavenging capacity. Results are summarized in Table 2. Novel compounds were, in general, more potent scavengers than reference compound Trolox (vitamin E analog). Interestingly, most of them exhibited a similar activity to melatonin being 2-folds more potent than Trolox, except compounds 11 and 12 which showed no scavenging capacity. Considering substituents, we can conclude that compounds bearing a $p$-tolyl substituent were the poorest scavenger of their corresponding subfamily (i.e., compounds 12, 15, and 18 from single acetylene, double acetylene and amide derivatives bearing an acetylene moiety, respectively). Importantly, reference compound rasagiline showed no effect in this assay.

Table 2. Antioxidant effect, anti-inflammatory properties, and BBB permeation capacity of compounds 10-18 and reference compounds. Free radical scavenging capacity was calculated from dose-response curves evaluating their ability to reduce ROS derived free radicals in the ORAC assay. Nitrite production reduction and IL-1 $\beta$ cytokine levels were evaluated in primary mixed glial cultures stimulated with LPS $(1 \mu \mathrm{g} / \mathrm{mL})$ for $18 \mathrm{~h}$ evaluated by the Griess method and ELISA, respectively. $\mathrm{EC}_{50}$ values for nitrite reduction were calculated from dose response curves using sulforaphane as positive control. BBB permeability was evaluated by the PAMPA assay. Data are expressed as mean \pm SEM of at least 3 independent experiments.

\begin{tabular}{|c|c|c|c|c|c|}
\hline \multirow{2}{*}{ Compound } & \multirow{2}{*}{$\begin{array}{l}\text { ORAC } \\
\text { (T. eq.) }\end{array}$} & \multirow{2}{*}{$\begin{array}{c}\mathrm{EC}_{50} \text { Nitrite } \\
\text { Reduction } \\
(\mu \mathrm{M})\end{array}$} & \multirow{2}{*}{$\begin{array}{c}\text { IL-1 } \beta \text { Levels } \\
\text { \% Reduction } \\
(10 \mu \mathrm{M})\end{array}$} & \multicolumn{2}{|c|}{ PAMPA } \\
\hline & & & & $\begin{array}{c}\mathrm{Pe} \\
\left(10^{-6} \mathrm{~cm} \mathrm{~s}^{-1}\right)\end{array}$ & Prediction \\
\hline Melatonin & $2.83 \pm 0.18$ & $25.7 \pm 2.5$ & $31.6 \pm 7.00$ & $\mathrm{NE}$ & \\
\hline Rasagiline & $0.04 \pm 0.02$ & NE & NE & $23.5 \pm 4.20$ & $\mathrm{CNS}+$ \\
\hline Sulforaphane & NE & $1.40 \pm 0.30[78]$ & $\mathrm{NE}$ & NE & NE \\
\hline 10 & $2.47 \pm 0.13$ & $16.2 \pm 2.50$ & $68.4 \pm 4.90$ & $12.3 \pm 0.62$ & $\mathrm{CNS}+$ \\
\hline 11 & $0.54 \pm 0.07$ & VR & VR & $5.08 \pm 1.70$ & $\mathrm{CNS}+$ \\
\hline 12 & $0.13 \pm 0.01$ & VR & VR & $1.84 \pm 0.48$ & $\mathrm{CNS}-$ \\
\hline 13 & $2.09 \pm 0.11$ & $12.6 \pm 0.53$ & $67.4 \pm 5.80$ & $20.3 \pm 7.50$ & $\mathrm{CNS}+$ \\
\hline 14 & $1.98 \pm 0.15$ & $19.4 \pm 1.40$ & $41.1 \pm 1.70$ & $4.15 \pm 1.80$ & $\mathrm{CNS}+$ \\
\hline 15 & $1.74 \pm 0.10$ & $22.9 \pm 3.30$ & $60.5 \pm 3.50$ & $4.46 \pm 1.90$ & $\mathrm{CNS}+$ \\
\hline 16 & $2.67 \pm 0.14$ & VR & VR & $6.64 \pm 1.30$ & $\mathrm{CNS}+$ \\
\hline 17 & $2.27 \pm 0.08$ & VR & VR & $3.12 \pm 1.20$ & $\mathrm{CNS} \pm$ \\
\hline 18 & $1.42 \pm 0.13$ & VR & VR & $2.04 \pm 3.20$ & $\mathrm{CNS} \pm$ \\
\hline
\end{tabular}

NA: not active; NE: not evaluated; VR: high cell viability reduction observed. CNS + (high BBB permeability): Pe $\left(10^{-6} \mathrm{~cm} \mathrm{~s}^{-1}\right)>4.0$; CNS-(low BBB permeability): Pe $\left(10^{-6} \mathrm{~cm} \mathrm{~s}^{-1}\right)<2.0$; CNS \pm (BBB permeability uncertain): $\mathrm{Pe}\left(10^{-6} \mathrm{~cm} \mathrm{~s}^{-1}\right)$ between 4.0 and $2.0[60]$.

Neuroinflammation has been demonstrated to be an essential contributor to PD pathogenesis, being a prominent feature of the disease $[79,80]$. Regarding the role of NRF2 in controlling several anti-inflammatory proteins [21] and the activation of this target elicited by novel compounds, we next evaluated the potential anti-inflammatory properties of compounds 10-18 in LPS stimulated primary mixed glial cultures. LPS binds to toll-like receptors 2 and 4 (TLR2 and TLR4) leading to nuclear factor- $\kappa \mathrm{B}$ (NF- $\kappa \mathrm{B}$ ) signaling activation. NF- $\kappa B$ promotes the expression of different pro-inflammatory genes such as inducible nitric oxide synthase (iNOS), NLR family pyrin domain containing 3 (NLRP3), and prointerleukin 1 beta (IL-1 $\beta$ ), among others. Mature IL-1 $\beta$ also signals via TLRs to further 
activate NF-кB [81]. iNOS enzyme over activation increases nitric oxide production and, therefore, nitrite levels. In this model, LPS promotes microglia and astrocytes polarization to a pro-inflammatory state, similarly to disease conditions [82,83]. Novel compounds showed interesting anti-inflammatory properties with $\mathrm{EC}_{50}$ values for nitrite reduction ranging from $12.6 \pm 0.53 \mu \mathrm{M}$ of compound 13 to $22.9 \pm 3.3 \mu \mathrm{M}$ of compound 15 (Table 2 ). Nonetheless, amide derivatives showed significant toxicity in our model, in line with high electrophilic character of this moiety and its potential off-target effects.

To further demonstrate the anti-inflammatory properties of our compounds, we next evaluated the levels of pro-inflammatory cytokine IL-1 $\beta$ in the same model. As shown in Table 2, compounds were able to reduce IL- $1 \beta$ release at $10 \mu \mathrm{M}$ concentration, exhibiting potencies from $41.1 \pm 1.7 \%$ of reduction by compound 14 to $68.4 \pm 4.9 \%$ of reduction by compound 10. Importantly, there is a cross-talk between NRF2 and NF- $\mathrm{K} B$ response pathways, interplaying at several levels. In general, NRF2 pathway activation decreases NF- $\kappa$ B response [84]. Thus, the anti-inflammatory properties of novel derivatives might be related to their NRF2 induction capacity. Similar properties were demonstrated for other NRF2 inducers including sulforaphane, able to reduce cyclooxygenase 2 expression, tumor necrosis factor alpha (TNF $\alpha)$, iNOS, and IL-1 $\beta$ in response to LPS in macrophages $[85,86]$.

Finally, considering their potential use for PD treatment, we explored their bloodbrain barrier (BBB) permeability prediction by passive diffusion of compounds $\mathbf{1 0 - 1 8}$ in the PAMPA assay. Results depicted in Table 2 indicate that all compounds are predicted to cross the BBB by passive diffusion with permeability $\left(\mathrm{P}_{\mathrm{e}}\right)$ values higher than $4.0 \cdot 10^{-6} \mathrm{~cm} \mathrm{~s}^{-1}$, except compound 12 that showed a low $\mathrm{P}_{\mathrm{e}}$ value. Compounds $\mathbf{1 7}$ and $\mathbf{1 8}$ showed uncertain BBB permeability with $\mathrm{P}_{\mathrm{e}}$ between 4.0 and $2.0 \cdot 10^{-6} \mathrm{~cm} \mathrm{~s}^{-1}$. Results obtained for reference compounds are shown in Table S3 (Supplementary Data).

\subsubsection{Neuroprotective Capacity of Compounds 10-18 in Oxidative Stress-Related Models}

As introduced, OS is one of the main events leading to neurodegeneration in PD [1]. Exacerbated OS status has been widely demonstrated in ex vivo samples of PD patients and associated to mitochondrial dysfunction [87]. Importantly, mutations in antioxidant-related genes such as PD protein 7 (PARK7) are associated with autosomal PD and directly correlate with increased cellular OS [88]. Additionally, as previously discussed, nigral dopaminergic neurons are highly vulnerable to OS due to dopamine metabolism by MAO enzymes. Therefore, we selected two in vitro models related to OS to evaluate the neuroprotective potential of compounds $\mathbf{1 0 - 1 8}$ in the human neuroblastoma cell line SH-SY5Y as shown in Figure $1 \mathrm{~A}$.

Firstly, we used the rotenone/oligomycin A toxic mixture as a model of mitochondrial dysfunction to induce OS-related toxicity. Rotenone is a mitochondrial complex I inhibitor and oligomycin A is an ATP synthase or complex V inhibitor [89]. This toxic combination impairs mitochondrial respiratory chain and produces a pro-oxidant environment in the cell similar to that observed in PD. Pre- and co-treatment with compounds 10-18 at a concentration of $0.1 \mu \mathrm{M}$ significantly protected cells from the toxic stimulus (Figure 1B). Interestingly, all the compounds showed percentages of neuroprotection higher than the reference compounds melatonin and rasagiline ranging from $32.1 \%$ exerted by compound 18 to $53.5 \%$ by compound 12 (Supplementary Data; Table S4). 
A

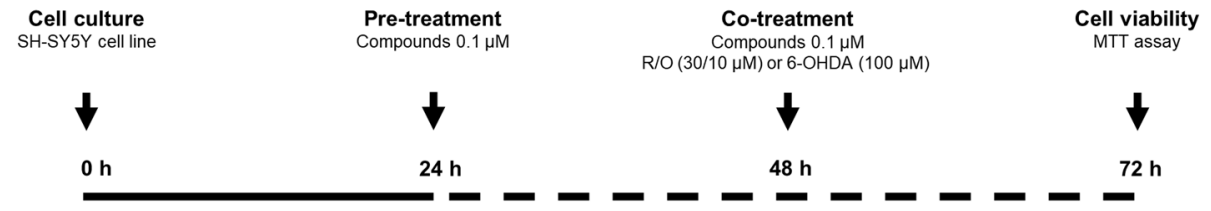

B

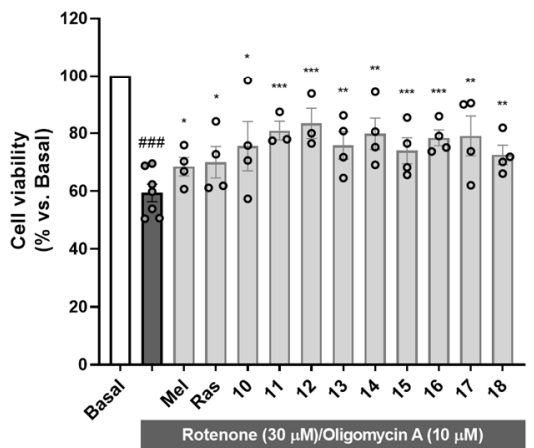

C

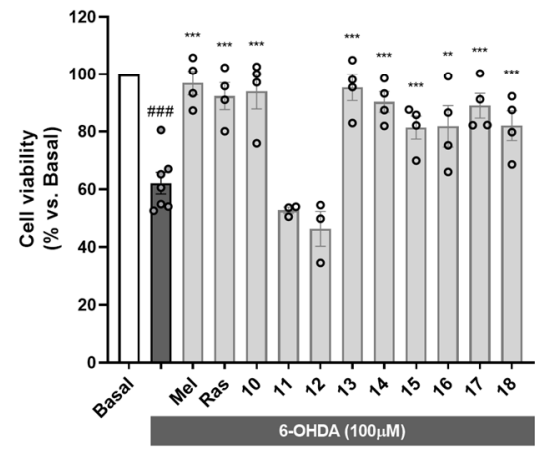

Figure 1. Neuroprotective activity of compounds 10-18 against the toxicity exerted by oxidative stressrelated toxic stimuli rotenone/oligomycin A mixture and 6-hydroxydopamine. (A) Experimental protocol scheme. SH-SY5Y cells were treated with compounds 10-18 $(0.1 \mu \mathrm{M})$ or reference compounds (melatonin and rasagiline, $0.1 \mu \mathrm{M}$ ) during $24 \mathrm{~h}$. Thereafter, cells were treated with compounds 10-18, melatonin or rasagiline, and the corresponding toxic stimuli: (B) R/O mixture $(30 / 10 \mu \mathrm{M})$ or $(\mathbf{C})$ 6-OHDA $(100 \mu \mathrm{M})$ for $24 \mathrm{~h}$. Cell viability was assessed by the MTT assay. Data are expressed as mean \pm SEM of 3-4 independent experiments. Statistical analysis was performed following one-way ANOVA $(p<0.05)$. \#\#\# $p<0.001$ vs. basal condition, ${ }^{*} p<0.033,{ }^{* *} p<0.002$ and ${ }^{* * *} p<0.001$ vs. the toxic condition after Newman-Keuls post hoc test.

Considering dopamine metabolism as a source of oxidative stress, 6-OHDA is a highly valuable model to reproduce PD pathology. This neurotoxin uses the dopamine transporter to selectively damage dopaminergic neurons. 6-OHDA toxicity is mainly related to its autoxidation leading to exacerbated OS and direct inhibition of mitochondrial respiratory chain complex I, among other events including microglial activation [90]. SH-SY5Y cells possess many characteristics of dopaminergic neurons, expressing tyrosine hydroxylase, dopamine-beta-hydroxylase and dopamine transporter [91]. Similarly to the previous model, pre- and co-treatment with compounds 10-18 at a concentration of $0.1 \mu \mathrm{M}$ led to significant neuroprotection, except for compounds $\mathbf{1 1}$ and $\mathbf{1 2}$ (Figure 1C). Treatment with the rest of the novel compounds almost completely rescued the cells from the toxic stimulus, with neuroprotection percentages ranging from $41.5 \%$ exerted by compound 15 to $87.4 \%$ of compound 13 (Supplementary Data; Table S4). Interestingly, the absence of protection observed with compounds 11 and 12 correlates with ORAC results where these compounds were inactive. These results are in line with the strong implication of autoxidation mechanisms in the toxicity elicited by 6-OHDA. In fact, melatonin showed the highest neuroprotection in this model $(88.8 \%)$. Nevertheless, rasagiline showed a strong protective capacity in this model $(77.0 \%)$, but no activity in the ORAC assay. This could be explained by its potent MAO inhibition. Like dopamine, 6-OHDA is processed by MAO enzymes, generating ROS [92]; thus, rasagiline could relieve 6-OHDA toxicity by inhibiting MAO-B, but also MAO-A in a medium nanomolar range $\left(\mathrm{IC}_{50}=4.43 \mathrm{nM}\right.$ for MAO-B compared to $\mathrm{IC}_{50}=412 \mathrm{nM}$ for MAO-A [93]). This is important considering that MAO metabolism in SH-SY5Y cells is mainly performed by MAO-A isozyme [94].

\subsubsection{Compound 14 Upregulates NRF2-Dependet Proteins}

After concluding the preliminary pharmacological screening, we aimed to obtain deeper insights into NRF2 activation mechanism. 
Compound 14 was selected as a hit compound considering its overall pharmacologic profile. Briefly, compound $\mathbf{1 4}$ was selected as, among our selective MAO-B inhibitors showing anti-inflammatory properties (i.e., compounds 13, 14, and 15), it was the most potent MAO-B inhibitor and showed potent NRF2 induction capability. Thus, to evaluate its mechanism of action, we measured firstly its capacity to upregulate the phase II antioxidant response. A dose-response curve for NRF2 induction in AREc32 cells is shown in Figure 2A. This result verifies that compound 14 induces NRF2, significantly increasing luciferase activity at 3, 5, and $10 \mu \mathrm{M}$. Thereafter, we used WT and NRF2 KO MEF cells to demonstrate a direct activation of the pathway following the protocol detailed in Figure 2B. Upon treatment with compound $14(10 \mu \mathrm{M})$ or sulforaphane $(10 \mu \mathrm{M})$ as positive control, cells were collected at different times to evaluate different proteins. Successfully, compound 14 increased NRF2-dependent proteins the levels of HMOX1 and GCLC in WT MEF cells (Figure 2C-G). In contrast, compound 14 was not able to increase them in NRF2 KO MEF cells confirming that it exerts its activity through NRF2 activation. Interestingly, HMOX1 and GCLC protein levels increase were time-dependent, as both proteins were further accumulated after $16 \mathrm{~h}$ treatment (Figure 2D,F) compared to $8 \mathrm{~h}$ (Figure 2E,G). Similar results were observed with the positive control, sulforaphane.

\subsubsection{Structural Basis for MAO-B Inhibition}

In order to study the structural mechanisms for MAO-B inhibition of our novel compounds that could lead to further optimization programs, we performed a computational study. For that purpose, we employed docking calculations followed by a 300 ns MD simulation, considering a reversible binding mode based on structural analogy of novel derivatives with other non-covalent inhibitors. We selected a crystal structure of human MAO-B with an active site open conformation (PDB-ID: 2BK3) [95], as our compounds are not expected to fit only in the reactive site cavity close to the FAD cofactor. This is an important assumption since MAO-B isozyme presents two cavities: an entry cavity and a reactive site or substrate cavity, separated by the "gating" residues Ile199 and Tyr326 [96]. In Figure 3A, we show the final and stabilized position of hit compound 14 at MAO-B active site after molecular dynamics simulation. It exhibited a binding mode along the bipartite pocket occupying both entrance and substrate cavities, a result that is frequently observed for large compounds as safinamide [97]. This class of compounds induce an open conformation of Ile199 side chain that, in our case, it is maintained during all MD simulation. Importantly, compound $\mathbf{1 4}$ proved to be able to stablish interactions with key enzyme residues. As shown in Figure 3A, it displayed hydrogen bonding with Pro102 residue and $\pi-\pi$ stacking interactions with Trp119, Phe168, Tyr326, and Phe343. Interestingly, Tyr326 and Ile199 are described as a key residues for inhibitor recognition and selectivity, as double Ile199Ala/Tyr326Ala mutations led to a protein exhibiting binding properties closer to $\mathrm{MAO}-\mathrm{A}[96]$. In that sense, the $\pi-\pi$ stacking interaction between the indole core of compound 14 and Tyr326 could be crucial for its activity and selectivity observed. Another significant finding is the fact that compound $\mathbf{1 4}$ was shown to locate the phenyl ring towards the aromatic cavity close to the FAD cofactor, interacting with important residues as Phe343, as seen for other potent and selective MAO-B inhibitors recently developed [98]. This aromatic cage is involved in catalysis and substrate specificity [99], thus, this interaction could be highly relevant for its inhibitory potency. In fact, aryl-acrylate moiety inclusion is related experimentally with higher inhibitory activities (Table 1). Similar binding modes were observed for all novel compounds (data not shown). 
A

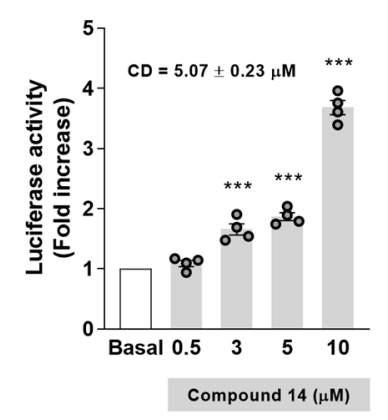

C

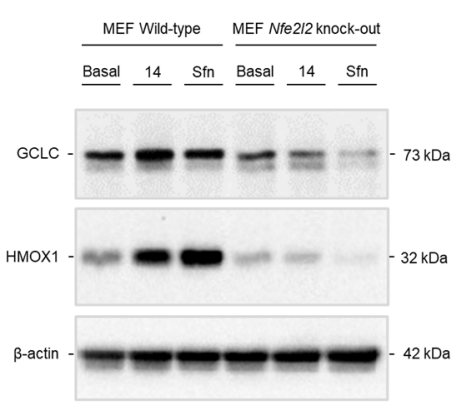

D
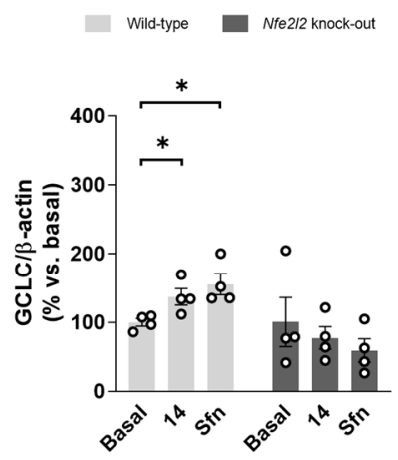

$\mathbf{F}$

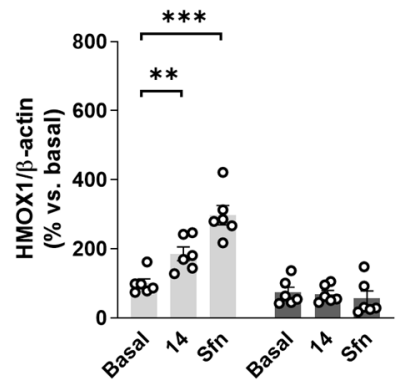

B

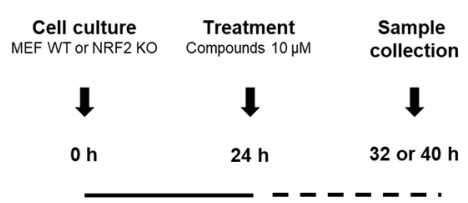

16 hours

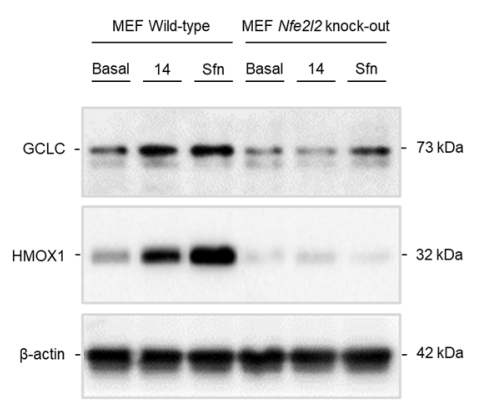

E
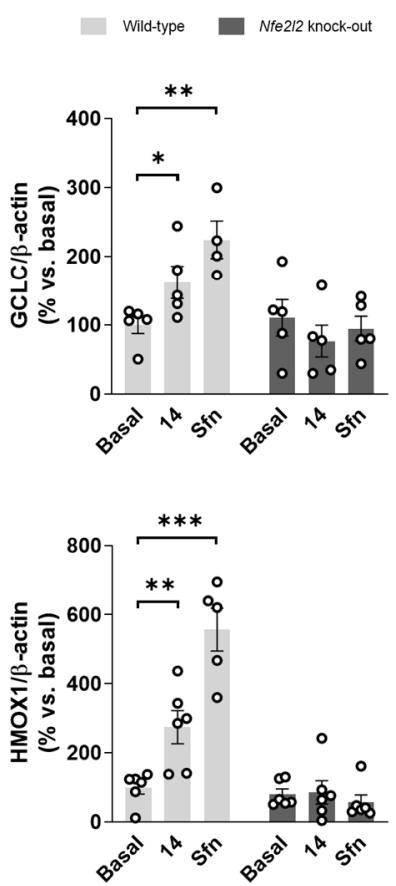

Figure 2. Compound 14 activates the phase II antioxidant response via NRF2 induction. (A) Doseresponse curve in AREc32 cells treated with compound $\mathbf{1 4}$ for $24 \mathrm{~h}$ calculated as luciferase activity normalized to basal conditions. (B) Experimental protocol followed to evaluate NRF2-related proteins induction. WT or NRF2 KO MEF cells were treated with compound $14(10 \mu \mathrm{M})$ or sulforaphane $(10 \mu \mathrm{M}$, positive control) across 8 or $16 \mathrm{~h}$. Thereafter, cells were processed for protein levels analysis. (C) Representative immunoblots. (D,E) Quantification of GCLC protein levels for WT and NRF2 KO MEF cells at 8 and $16 \mathrm{~h}$, respectively. (F,G) Quantification of HMOX1 protein levels for WT and NRF2 KO MEF at 8 and $16 \mathrm{~h}$, respectively. Data are expressed as mean \pm SEM of at least 4 independent experiments. Comparisons were made using one-way ANOVA test $(p<0.05)$ followed by the Newman-Keuls post hoc analysis (A) or an unpaired $t$-test (D-G). ${ }^{*} p<0.033,{ }^{* *} p<0.002$, and $* * * * 0.001$. 


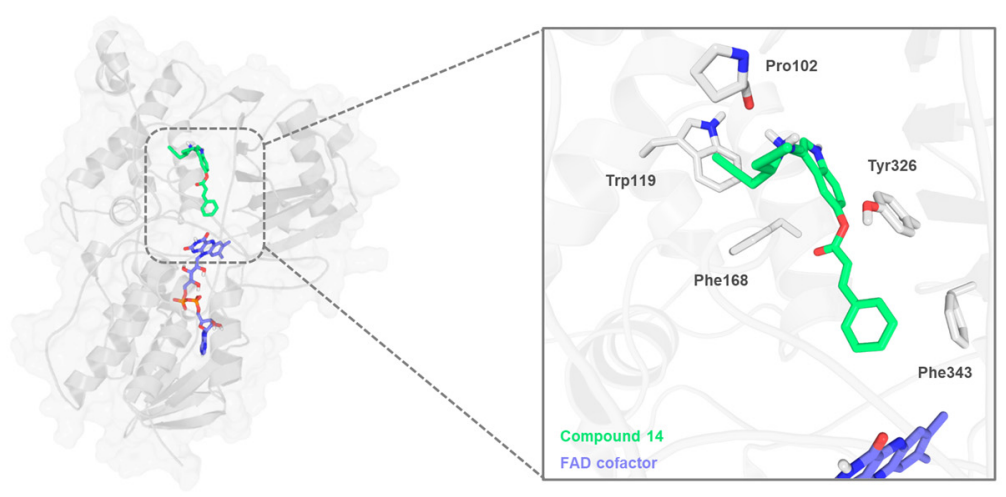

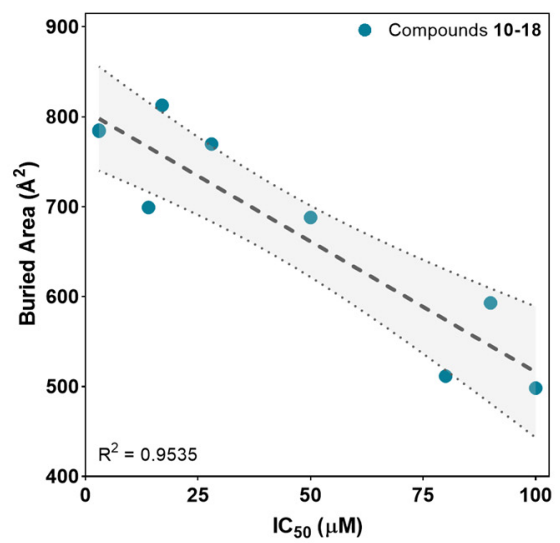

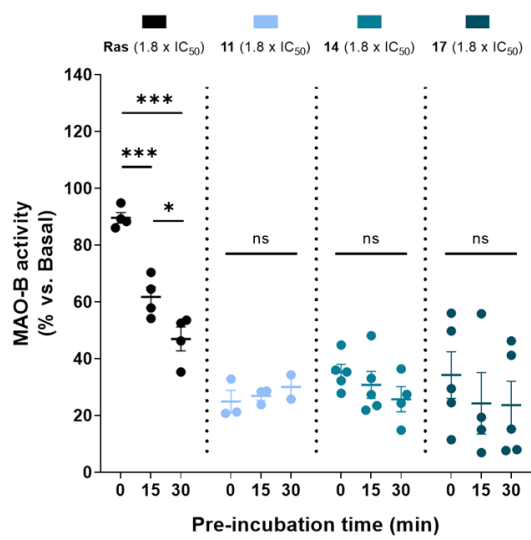

C

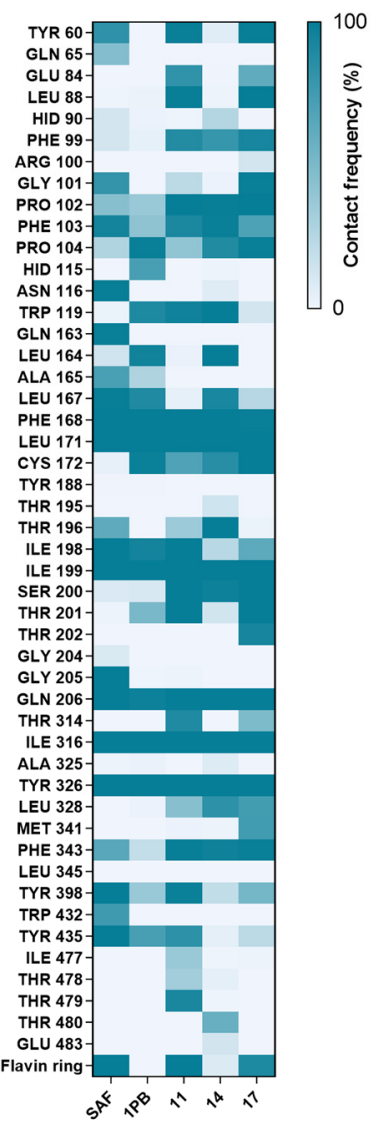

Figure 3. MAO-B binding mode determination. (A) Left, global view of the predicted conformation of hit compound 14 in complex with MAO-B after docking and 300 ns molecular dynamics simulation. Right, detailed position at the active site with key protein residues involved in the interaction with the ligand represented as colored sticks. MAO-B protein is represented as gray cartoon and surface. (B) Correlation between experimental $\mathrm{IC}_{50}$ values for MAO-B inhibition and buried area for proteinligand interaction. Dotted gray line indicates the linear regression ( $\pm 95 \%$ confidence interval). (C) Protein-ligand contact frequency map of safinamide, 1,4-diphenyl-2-butene, and compounds 11, 14, and 17 at the active site. Colors represent the percentage of frames in which there is a distance below $5 \AA$ A between a certain residue and the ligand. Results were calculated for the 300 ns simulation. (D) Reversibility assay for studying MAO-B kinetics. MAO-B activity was measured after different ligand-enzyme pre-incubation times at $1.8 \times \mathrm{IC}_{50} \mu \mathrm{M}$ concentration. Data are expressed as mean \pm SEM of at least 3 independent experiments. Comparisons were made using one-way ANOVA test $(p<0.05)$ followed by Newman-Keuls post hoc analysis. ${ }^{*} p<0.033$ and ${ }^{* * *} p<0.001$. 
We then calculated the buried area for compounds 10-18, which measures the size of the interface in the protein-ligand complex. Higher values of this parameter indicate higher interaction surface between tested compound and the protein being considered as a better ligand stabilization at the active site. As represented in Figure 3B, there is a correlation between the buried area and experimental $\mathrm{IC}_{50}$ values for MAO-B inhibition. This result indicates that our model could be further used for activity predictions. We also analyzed the contact frequency between ligands and relevant residues at the active site of the enzyme. The resulting contact map is shown in Figure $3 \mathrm{C}$ for a representative compound of each subfamily (i.e., compounds 11, 14, and 17) and known inhibitors safinamide and 1,4-diphenyl-2-butene. 1,4-diphenyl-2-butene, which inhibits MAO-B in the same range as our compounds $(\mathrm{Ki}=35 \mu \mathrm{M})$ [100], did not show relevant additional residue interactions compared to them, again validating the model. Considering safinamide as reference $(\mathrm{Ki}=450 \mathrm{nM})$ [97], we can see slight changes in contacts that could explain the activity variations. As shown in Figure 3C, we can highlight safinamide interactions with Gln65, Asn116, Gln163, Gly205, and Trp432 that are absent in compounds 11, 14, and 17. In contrast, they display some interactions at a specific region of the entrance cavity (Glu84, Leu88, and Phe99) that might be detrimental for their inhibitory capacity, considering that this area is far from the reactive site.

Finally, we performed a reversibility test to evaluate their binding kinetics. This is based on the use of different pre-incubation times of compounds with MAO-B enzyme prior to measure the enzymatic activity. Irreversible inhibitors as rasagiline show decreased activity over time, since they have more time to inhibit the enzyme by covalent bonding. In contrast, MAO-B activity remains time-invariant upon treatment with reversible inhibitors. Results are depicted in Figure 3D, showing a reversible inhibition elicited by representative compounds of each subfamily (i.e., compounds 11, 14, and 17) compared to rasagiline.

3.2.6. Compound 14 Reduces Cell Death and Oxidative Stress Production in Rat Striatal Slices as an Acute Ex Vivo Model of PD

Finally, we planned to evaluate the neuroprotective and antioxidant properties of hit compound 14 in adult rat striatal slices treated with rotenone or 6-OHDA. This ex vivo model is considered a more complex experimental setup related to PD. As introduced, PD is characterized by neurodegeneration of the nigrostriatal pathway and striatum constitutes a brain region tightly related with the molecular events occurring during disease progression [1,101]. Previous studies have characterized the 6-OHDA [102-104] and rotenone [105] induced toxicity models in striatal slices. Here, we selected adult rat striatal slices subjected to acute 6-OHDA or rotenone toxicity as shown in Figure 4A. After slices dissection and stabilization, they were co-treated with 6-OHDA $(100 \mu \mathrm{M})$ or rotenone $(10 \mu \mathrm{M})$ and compound $14(10 \mu \mathrm{M})$ during $4 \mathrm{~h}$. Melatonin $(10 \mu \mathrm{M})$ was included for comparative purposes. As previously reported, 6-OHDA treatment significantly increased cell death and ROS production $[103,104]$. Compound 14 treatment led to a significant reduction in cell death measured with the fluorescent dye propidium iodide (Figure 4B,D). Moreover, compound 14 significantly decreased ROS production evaluated with the fluorescent probe $\mathrm{H}_{2} \mathrm{DCFDA}$ (Figure 4B,E). Interestingly, compound $\mathbf{1 4}$ had the same protective capacity as melatonin in the 6-OHDA model, completely protecting slices from the toxic stimulus. Previous studies had showed superoxide and nitric oxide increases in striatal slices upon treatment with rotenone [105]. In our model, rotenone treatment also led to an increase in ROS levels, which were reduced by compound $\mathbf{1 4}$ or melatonin treatments (Figure 4C,F). Cell death was not significantly altered in this model. Although our compounds were protective in the SH-SY5Y model, striatal slices constitute a more complex scenario. Glia contribution is important in this case, regarding its connection with neurotoxicity related to dopamine metabolism by MAO-B enzyme. Pharmacological profile of compound $\mathbf{1 4}$ is relevant in the context of the results obtained, regarding all the biological implications of its main targets. 


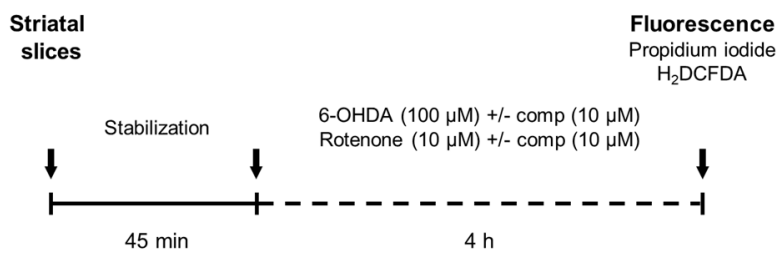

B

C
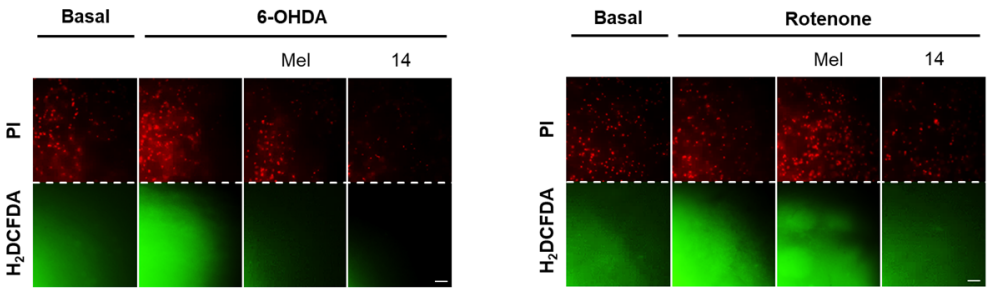

D

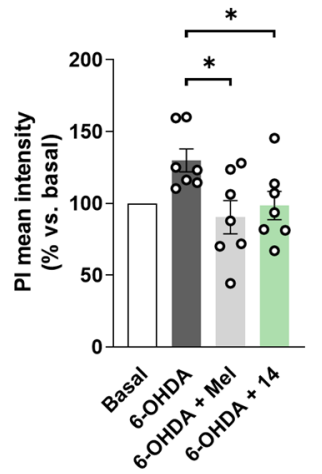

E

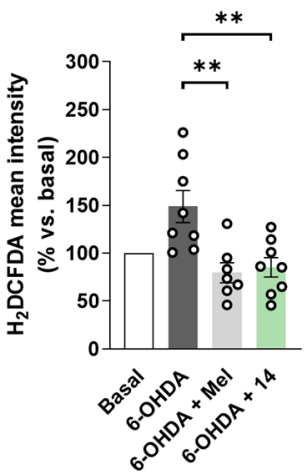

$\mathbf{F}$

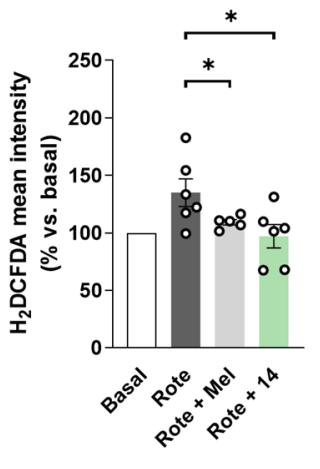

Figure 4. Compound 14 reduced cell death and restored ROS levels in rat striatal slices treated with 6-OHDA or rotenone. (A) Experimental protocol scheme. Striatal slices were obtained from adult rats. They were co-treated with compound $14(10 \mu \mathrm{M})$ and 6-OHDA $(100 \mu \mathrm{M})$ or rotenone $(10 \mu \mathrm{M})$ for $4 \mathrm{~h}$. Melatonin $(10 \mu \mathrm{M})$ was included for comparative purposes. $(\mathbf{B}, \mathbf{C})$ Representative fluorescence images for cell death (PI) and ROS production ( $\mathrm{H}_{2}$ DCFDA) for 6-OHDA and rotenone, respectively (Scale bar $=50 \mu \mathrm{m}$ ). Cell death quantification of $(\mathbf{D})$ and ROS production $(\mathrm{E})$ in the 6-OHDA model. (F) ROS production quantification in the rotenone model. Data are expressed as mean $\pm \mathrm{SEM}$ of at least 5 independent experiments. Comparisons were made using one-way ANOVA test $(p<0.05)$ followed by Newman-Keuls post hoc analysis. ${ }^{*} p<0.033$ and ${ }^{* *} p<0.002$.

\section{Conclusions}

The novel 2-(1H-indol-3-yl)ethan-1-amine derivatives 10-18 were designed as novel NRF2 inducers and selective MAO-B inhibitors for PD treatment. Pharmacological screening of these compounds proved that they were active for their main targets. In that sense, compounds 10-18 showed, in general, NRF2 induction activity and selective MAO-B inhibition at a low-to-medium micromolar range. Additionally, they exerted ROS scavenging capacity, similar to their parental compound melatonin, and anti-inflammatory properties in primary mixed glial cultures in terms of nitrites and IL- $1 \beta$ reduction upon LPS stimulation. Compounds $\mathbf{1 0 - 1 8}$ were in general predicted to reach the central nervous system evaluated by PAMPA assay and they were protective in oxidative stress-related models in SH-SY5Y cells. We could stablish structure-activity relationships that explain the potency variations observed in the in vitro experiments. In light of these results, we selected compound $\mathbf{1 4}$ as the hit compound based on its overall pharmacological profile to obtain deeper insights into its mechanisms of action. Compound 14 effectively upregulated NRF2-dependent proteins and we confirmed with MEF cells obtained from NRF2 WT and 
KO mice that this effect was exerted through NRF2 activation. Importantly, this compound reduced cell death and reestablished ROS levels in a more complex model in rat striatal slices treated with 6-OHDA or rotenone. Additionally, computational data allowed us to describe its predicted binding mode at the MAO-B active site, drawing important conclusions for further optimization programs. Our novel approach combining NRF2 induction with selective MAO-B inhibition, exemplified by hit compound 14, could lead future efforts towards the development of a disease-modifying drug candidate for PD treatment.

\section{Patents}

The results here described have been protected in patent application P202230025. Priority date: 14/01/2022.

Supplementary Materials: The following are available online at https:/ / www.mdpi.com/article/10 .3390/antiox11020247/s1, Table S1: Physicochemical properties; Table S2: Cytotoxicity elicited by compounds 10-18 in the SH-SY5Y, AREc32 cell lines and primary glial cells; Table S3: Prediction of the BBB passive permeability of control compounds; Table S4: Neuroprotective activity of compounds 10-18. Original WB images; Figure S1: RMSD calculations of molecular dynamic simulations; copies of NMR spectra.

Author Contributions: P.D. carried out the chemistry, performed biological, computational experiments and wrote the paper. P.M. carried out the chemistry and performed biological experiments. E.C. performed computational experiments. A.C. supervised the project and critically revised the manuscript. R.L. designed the project, supervised experiments, and wrote the paper. All authors have read and agreed to the published version of the manuscript.

Funding: This research was funded by the IS Carlos III co-financed by the European Regional Development funds (FEDER) (grant PI17/01700 to RL) by the Spanish Ministry of Economy and Competitiveness (MINECO) (grant PID2019-110061RB-I00 to A.C.) and Comunidad Autónoma de Madrid (grant B2017/BMD-3827, RL). The APC was not funded.

Institutional Review Board Statement: All experimental procedures were performed following the Guide for the Care and Use of Laboratory Animals, in accordance with the European Guidelines for the use and care of animals for research in accordance with the European Union Directive of 22 Sep-tember 2010 (2010/63/UE) and with the Spanish Royal Decree of 1 February 2013 (53/2013).

Informed Consent Statement: Not applicable.

Data Availability Statement: Data are contained within the article or Supplementary Materials.

Acknowledgments: P.D. and P.M. thank MECD for FPU fellowships (16/03977, and 13/03737, respectively). E.C. thank IS Carlos III for PFIS fellowship (FI18/00306). This article is based upon work from COST Action CA20121, supported by COST (European Cooperation in Science and Technology) (www.cost.eu, accessed 20 December 2021). Authors acknowledge Instituto Teófilo Hernando for its continued support.

Conflicts of Interest: The authors declare no conflict of interest.

\section{References}

1. Poewe, W.; Seppi, K.; Tanner, C.M.; Halliday, G.M.; Brundin, P.; Volkmann, J.; Schrag, A.E.; Lang, A.E. Parkinson disease. Nat. Rev. Dis. Primers 2017, 3, 17013. [CrossRef]

2. Cieri, D.; Brini, M.; Calì, T. Emerging (and converging) pathways in Parkinson's disease: Keeping mitochondrial wellness. Biochem. Biophys. Res. Commun. 2017, 483, 1020-1030. [CrossRef]

3. Lang, A.E.; Lozano, A.M. Parkinson's disease. First of two parts. N. Engl. J. Med. 1998, 339, 1044-1053. [CrossRef]

4. Wakabayashi, K.; Tanji, K.; Mori, F.; Takahashi, H. The Lewy body in Parkinson's disease: Molecules implicated in the formation and degradation of alpha-synuclein aggregates. Neuropathology 2007, 27, 494-506. [CrossRef]

5. Yacoubian, T.A.; Standaert, D.G. Targets for neuroprotection in Parkinson's disease. Biochim. Biophys. Acta 2009, 1792, 676-687. [CrossRef]

6. Duarte, P.; Cuadrado, A.; León, R. Monoamine Oxidase Inhibitors: From Classic to New Clinical Approaches. Handb. Exp. Pharmacol. 2021, 264, 229-259. [CrossRef]

7. Segura-Aguilar, J.; Paris, I.; Munoz, P.; Ferrari, E.; Zecca, L.; Zucca, F.A. Protective and toxic roles of dopamine in Parkinson's disease. J. Neurochem. 2014, 129, 898-915. [CrossRef] 
8. Fowler, J.S.; Volkow, N.D.; Wang, G.J.; Logan, J.; Pappas, N.; Shea, C.; MacGregor, R. Age-related increases in brain monoamine oxidase B in living healthy human subjects. Neurobiol. Aging 1997, 18, 431-435. [CrossRef]

9. Nagatsu, T.; Sawada, M. Molecular mechanism of the relation of monoamine oxidase B and its inhibitors to Parkinson's disease: Possible implications of glial cells. J. Neural Transmission. Suppl. 2006, 71, 53-65. [CrossRef]

10. Mallajosyula, J.K.; Kaur, D.; Chinta, S.J.; Rajagopalan, S.; Rane, A.; Nicholls, D.G.; Di Monte, D.A.; Macarthur, H.; Andersen, J.K. MAO-B elevation in mouse brain astrocytes results in Parkinson's pathology. PLoS ONE 2008, 3, e1616. [CrossRef]

11. Sian, J.; Dexter, D.T.; Lees, A.J.; Daniel, S.; Agid, Y.; Javoy-Agid, F.; Jenner, P.; Marsden, C.D. Alterations in glutathione levels in Parkinson's disease and other neurodegenerative disorders affecting basal ganglia. Ann. Neurol. 1994, 36, 348-355. [CrossRef]

12. Jo, S.; Yarishkin, O.; Hwang, Y.J.; Chun, Y.E.; Park, M.; Woo, D.H.; Bae, J.Y.; Kim, T.; Lee, J.; Chun, H.; et al. GABA from reactive astrocytes impairs memory in mouse models of Alzheimer's disease. Nat. Med. 2014, 20, 886-896. [CrossRef]

13. Heo, J.Y.; Nam, M.H.; Yoon, H.H.; Kim, J.; Hwang, Y.J.; Won, W.; Woo, D.H.; Lee, J.A.; Park, H.J.; Jo, S.; et al. Aberrant Tonic Inhibition of Dopaminergic Neuronal Activity Causes Motor Symptoms in Animal Models of Parkinson's Disease. Curr. Biol. CB 2020, 30, 276-291. [CrossRef]

14. Nam, M.H.; Park, J.H.; Song, H.J.; Choi, J.W.; Kim, S.; Jang, B.K.; Yoon, H.H.; Heo, J.Y.; Lee, H.; An, H.; et al. KDS2010, a Newly Developed Reversible MAO-B Inhibitor, as an Effective Therapeutic Candidate for Parkinson's Disease. Neurother. J. Am. Soc. Exp. NeuroTher. 2021, 18, 1729-1747. [CrossRef]

15. Hayes, J.D.; Dinkova-Kostova, A.T. The Nrf2 regulatory network provides an interface between redox and intermediary metabolism. Trends Biochem. Sci. 2014, 39, 199-218. [CrossRef]

16. Buendia, I.; Michalska, P.; Navarro, E.; Gameiro, I.; Egea, J.; Leon, R. Nrf2-ARE pathway: An emerging target against oxidative stress and neuroinflammation in neurodegenerative diseases. Pharmacol. Ther. 2016, 157, 84-104. [CrossRef]

17. Pajares, M.; Jiménez-Moreno, N.; García-Yagüe, Á.J.; Escoll, M.; de Ceballos, M.L.; Van Leuven, F.; Rábano, A.; Yamamoto, M.; Rojo, A.I.; Cuadrado, A. Transcription factor NFE2L2/NRF2 is a regulator of macroautophagy genes. Autophagy 2016, 12, 1902-1916. [CrossRef]

18. Cuadrado, A.; Moreno-Murciano, P.; Pedraza-Chaverri, J. The transcription factor Nrf2 as a new therapeutic target in Parkinson's disease. Expert Opin. Ther. Targets 2009, 13, 319-329. [CrossRef]

19. Schipper, H.M.; Song, W.; Zukor, H.; Hascalovici, J.R.; Zeligman, D. Heme oxygenase-1 and neurodegeneration: Expanding frontiers of engagement. J. Neurochem. 2009, 110, 469-485. [CrossRef]

20. van Muiswinkel, F.L.; de Vos, R.A.; Bol, J.G.; Andringa, G.; Jansen Steur, E.N.; Ross, D.; Siegel, D.; Drukarch, B. Expression of $\mathrm{NAD}(\mathrm{P}) \mathrm{H}$ :quinone oxidoreductase in the normal and Parkinsonian substantia nigra. Neurobiol. Aging 2004, 25, 1253-1262. [CrossRef]

21. Cuadrado, A.; Rojo, A.I.; Wells, G.; Hayes, J.D.; Cousin, S.P.; Rumsey, W.L.; Attucks, O.C.; Franklin, S.; Levonen, A.L.; Kensler, T.W.; et al. Therapeutic targeting of the NRF2 and KEAP1 partnership in chronic diseases. Nat. Rev. Drug Discov. 2019, 18, 295-317. [CrossRef] [PubMed]

22. Lastres-Becker, I.; García-Yagüe, A.J.; Scannevin, R.H.; Casarejos, M.J.; Kügler, S.; Rábano, A.; Cuadrado, A. Repurposing the NRF2 Activator Dimethyl Fumarate as Therapy Against Synucleinopathy in Parkinson's Disease. Antioxid. Redox Signal. 2016, 25, 61-77. [CrossRef] [PubMed]

23. Ramsey, C.P.; Glass, C.A.; Montgomery, M.B.; Lindl, K.A.; Ritson, G.P.; Chia, L.A.; Hamilton, R.L.; Chu, C.T.; Jordan-Sciutto, K.L. Expression of Nrf2 in neurodegenerative diseases. J. Neuropathol. Exp. Neurol. 2007, 66, 75-85. [CrossRef] [PubMed]

24. Loeffler, D.A.; Smith, L.M.; Coffey, M.P.; Aasly, J.O.; LeWitt, P.A. CSF Nrf2 and HSPA8 in Parkinson's disease patients with and without LRRK2 gene mutations. J. Neural Transm. 2016, 123, 179-187. [CrossRef]

25. von Otter, M.; Bergström, P.; Quattrone, A.; De Marco, E.V.; Annesi, G.; Söderkvist, P.; Wettinger, S.B.; Drozdzik, M.; Bialecka, M.; Nissbrandt, H.; et al. Genetic associations of Nrf2-encoding NFE2L2 variants with Parkinson's disease-a multicenter study. BMC Med. Genet. 2014, 15, 131. [CrossRef]

26. von Otter, M.; Landgren, S.; Nilsson, S.; Celojevic, D.; Bergström, P.; Håkansson, A.; Nissbrandt, H.; Drozdzik, M.; Bialecka, M.; Kurzawski, M.; et al. Association of Nrf2-encoding NFE2L2 haplotypes with Parkinson's disease. BMC Med. Genet. 2010, 11, 36. [CrossRef]

27. Lastres-Becker, I.; Ulusoy, A.; Innamorato, N.G.; Sahin, G.; Rábano, A.; Kirik, D.; Cuadrado, A. $\alpha$-Synuclein expression and Nrf2 deficiency cooperate to aggravate protein aggregation, neuronal death and inflammation in early-stage Parkinson's disease. Hum. Mol. Genet. 2012, 21, 3173-3192. [CrossRef]

28. Gan, L.; Vargas, M.R.; Johnson, D.A.; Johnson, J.A. Astrocyte-specific overexpression of Nrf2 delays motor pathology and synuclein aggregation throughout the CNS in the alpha-synuclein mutant (A53T) mouse model. J. Neurosci. 2012, 32, 17775-17787. [CrossRef]

29. Jing, X.; Shi, H.; Zhang, C.; Ren, M.; Han, M.; Wei, X.; Zhang, X.; Lou, H. Dimethyl fumarate attenuates 6-OHDA-induced neurotoxicity in SH-SY5Y cells and in animal model of Parkinson's disease by enhancing Nrf2 activity. Neuroscience 2015, 286, 131-140. [CrossRef]

30. Jazwa, A.; Rojo, A.I.; Innamorato, N.G.; Hesse, M.; Fernández-Ruiz, J.; Cuadrado, A. Pharmacological targeting of the transcription factor Nrf2 at the basal ganglia provides disease modifying therapy for experimental parkinsonism. Antioxid. Redox Signal. 2011, 14, 2347-2360. [CrossRef] 
31. Kaidery, N.A.; Banerjee, R.; Yang, L.; Smirnova, N.A.; Hushpulian, D.M.; Liby, K.T.; Williams, C.R.; Yamamoto, M.; Kensler, T.W.; Ratan, R.R.; et al. Targeting Nrf2-mediated gene transcription by extremely potent synthetic triterpenoids attenuate dopaminergic neurotoxicity in the MPTP mouse model of Parkinson's disease. Antioxid. Redox Signal. 2013, 18, 139-157. [CrossRef] [PubMed]

32. Chen, P.C.; Vargas, M.R.; Pani, A.K.; Smeyne, R.J.; Johnson, D.A.; Kan, Y.W.; Johnson, J.A. Nrf2-mediated neuroprotection in the MPTP mouse model of Parkinson's disease: Critical role for the astrocyte. Proc. Natl. Acad. Sci. USA 2009, 106, $2933-2938$. [CrossRef] [PubMed]

33. Rojo, A.I.; Innamorato, N.G.; Martín-Moreno, A.M.; De Ceballos, M.L.; Yamamoto, M.; Cuadrado, A. Nrf2 regulates microglial dynamics and neuroinflammation in experimental Parkinson's disease. Glia 2010, 58, 588-598. [CrossRef] [PubMed]

34. Canning, P.; Sorrell, F.J.; Bullock, A.N. Structural basis of Keap1 interactions with Nrf2. Free. Radic. Biol. Med. 2015, 88, 101-107. [CrossRef]

35. Dinkova-Kostova, A.T.; Holtzclaw, W.D.; Cole, R.N.; Itoh, K.; Wakabayashi, N.; Katoh, Y.; Yamamoto, M.; Talalay, P. Direct evidence that sulfhydryl groups of Keap1 are the sensors regulating induction of phase 2 enzymes that protect against carcinogens and oxidants. Proc. Natl. Acad. Sci. USA 2002, 99, 11908-11913. [CrossRef]

36. Zhang, D.D.; Hannink, M. Distinct cysteine residues in Keap1 are required for Keap1-dependent ubiquitination of Nrf2 and for stabilization of Nrf2 by chemopreventive agents and oxidative stress. Mol. Cell. Biol. 2003, 23, 8137-8151. [CrossRef]

37. Brennan, M.S.; Matos, M.F.; Li, B.; Hronowski, X.; Gao, B.; Juhasz, P.; Rhodes, K.J.; Scannevin, R.H. Dimethyl fumarate and monoethyl fumarate exhibit differential effects on KEAP1, NRF2 activation, and glutathione depletion in vitro. PLoS ONE 2015, 10, e0120254. [CrossRef]

38. Tan, D.X.; Manchester, L.C.; Terron, M.P.; Flores, L.J.; Reiter, R.J. One molecule, many derivatives: A never-ending interaction of melatonin with reactive oxygen and nitrogen species? J. Pineal Res. 2007, 42, 28-42. [CrossRef]

39. Luchetti, F.; Canonico, B.; Betti, M.; Arcangeletti, M.; Pilolli, F.; Piroddi, M.; Canesi, L.; Papa, S.; Galli, F. Melatonin signaling and cell protection function. FASEB J. 2010, 24, 3603-3624. [CrossRef]

40. Pandi-Perumal, S.R.; BaHammam, A.S.; Brown, G.M.; Spence, D.W.; Bharti, V.K.; Kaur, C.; Hardeland, R.; Cardinali, D.P. Melatonin antioxidative defense: Therapeutical implications for aging and neurodegenerative processes. Neurotox. Res. 2013, 23, 267-300. [CrossRef]

41. Aguiar, L.M.; Vasconcelos, S.M.; Sousa, F.C.; Viana, G.S. Melatonin reverses neurochemical alterations induced by 6-OHDA in rat striatum. Life Sci. 2002, 70, 1041-1051. [CrossRef]

42. Ozsoy, O.; Yildirim, F.B.; Ogut, E.; Kaya, Y.; Tanriover, G.; Parlak, H.; Agar, A.; Aslan, M. Melatonin is protective against 6-hydroxydopamine-induced oxidative stress in a hemiparkinsonian rat model. Free. Radic. Res. 2015, 49, 1004-1014. [CrossRef] [PubMed]

43. Sharma, R.; McMillan, C.R.; Tenn, C.C.; Niles, L.P. Physiological neuroprotection by melatonin in a 6-hydroxydopamine model of Parkinson's disease. Brain Res. 2006, 1068, 230-236. [CrossRef] [PubMed]

44. Chen, S.T.; Chuang, J.I.; Hong, M.H.; Li, E.I. Melatonin attenuates MPP+-induced neurodegeneration and glutathione impairment in the nigrostriatal dopaminergic pathway. J. Pineal Res. 2002, 32, 262-269. [CrossRef] [PubMed]

45. López, A.; Ortiz, F.; Doerrier, C.; Venegas, C.; Fernández-Ortiz, M.; Aranda, P.; Díaz-Casado, M.E.; Fernández-Gil, B.; BarriocanalCasado, E.; Escames, G.; et al. Mitochondrial impairment and melatonin protection in parkinsonian mice do not depend of inducible or neuronal nitric oxide synthases. PLoS ONE 2017, 12, e0183090. [CrossRef]

46. Naskar, A.; Prabhakar, V.; Singh, R.; Dutta, D.; Mohanakumar, K.P. Melatonin enhances L-DOPA therapeutic effects, helps to reduce its dose, and protects dopaminergic neurons in 1-methyl-4-phenyl-1,2,3,6-tetrahydropyridine-induced Parkinsonism in mice. J. Pineal Res. 2015, 58, 262-274. [CrossRef]

47. Tapias, V.; Escames, G.; López, L.C.; López, A.; Camacho, E.; Carrión, M.D.; Entrena, A.; Gallo, M.A.; Espinosa, A.; AcuñaCastroviejo, D. Melatonin and its brain metabolite N(1)-acetyl-5-methoxykynuramine prevent mitochondrial nitric oxide synthase induction in parkinsonian mice. J. Neurosci. Res. 2009, 87, 3002-3010. [CrossRef]

48. Carriere, C.H.; Kang, N.H.; Niles, L.P. Chronic low-dose melatonin treatment maintains nigrostriatal integrity in an intrastriatal rotenone model of Parkinson's disease. Brain Res. 2016, 1633, 115-125. [CrossRef]

49. Saravanan, K.S.; Sindhu, K.M.; Mohanakumar, K.P. Melatonin protects against rotenone-induced oxidative stress in a hemiparkinsonian rat model. J. Pineal Res. 2007, 42, 247-253. [CrossRef]

50. Brito-Armas, J.M.; Baekelandt, V.; Castro-Hernández, J.R.; González-Hernández, T.; Rodríguez, M.; Castro, R. Melatonin prevents dopaminergic cell loss induced by lentiviral vectors expressing A30P mutant alpha-synuclein. Histol. Histopathol. 2013, 28, 999-1006. [CrossRef]

51. Ahn, J.H.; Kim, M.; Park, S.; Jang, W.; Park, J.; Oh, E.; Cho, J.W.; Kim, J.S.; Youn, J. Prolonged-release melatonin in Parkinson's disease patients with a poor sleep quality: A randomized trial. Parkinsonism Relat. Disord. 2020, 75, 50-54. [CrossRef] [PubMed]

52. Daneshvar Kakhaki, R.; Ostadmohammadi, V.; Kouchaki, E.; Aghadavod, E.; Bahmani, F.; Tamtaji, O.R.; Reiter, J.R.; Mansournia, M.A.; Asemi, Z. Melatonin supplementation and the effects on clinical and metabolic status in Parkinson's disease: A randomized, double-blind, placebo-controlled trial. Clin. Neurol. Neurosurg. 2020, 195, 105878. [CrossRef] [PubMed]

53. Delgado-Lara, D.L.; González-Enríquez, G.V.; Torres-Mendoza, B.M.; González-Usigli, H.; Cárdenas-Bedoya, J.; Macías-Islas, M.A.; de la Rosa, A.C.; Jiménez-Delgado, A.; Pacheco-Moisés, F.; Cruz-Serrano, J.A.; et al. Effect of melatonin administration on the PER1 and BMAL1 clock genes in patients with Parkinson's disease. Biomed. Pharmacother. Biomed. Pharmacother. 2020, 129, 110485. [CrossRef] [PubMed] 
54. Dowling, G.A.; Mastick, J.; Colling, E.; Carter, J.H.; Singer, C.M.; Aminoff, M.J. Melatonin for sleep disturbances in Parkinson's disease. Sleep Med. 2005, 6, 459-466. [CrossRef]

55. Litvinenko, I.V.; Krasakov, I.V.; Tikhomirova, O.V. Sleep disorders in Parkinson's disease without dementia: A comparative randomized controlled study of melatonin and clonazepam. Zhurnal Nevrol. I Psikhiatrii Im. S.S. Korsakova 2012, 112, 26-30.

56. Medeiros, C.A.; Carvalhedo de Bruin, P.F.; Lopes, L.A.; Magalhães, M.C.; de Lourdes Seabra, M.; de Bruin, V.M. Effect of exogenous melatonin on sleep and motor dysfunction in Parkinson's disease. A randomized, double blind, placebo-controlled study. J. Neurol. 2007, 254, 459-464. [CrossRef]

57. Ortiz, G.G.; Moráles-Sánchez, E.W.; Pacheco-Moisés, F.P.; Jiménez-Gil, F.J.; Macías-Islas, M.A.; Mireles-Ramírez, M.A.; GonzálezUsigli, H. Effect of melatonin administration on cyclooxygenase-2 activity, serum levels of nitric oxide metabolites, lipoperoxides and glutathione peroxidase activity in patients with Parkinson's disease. Gac. Med. Mex. 2017, 153, S72-S81. [CrossRef]

58. Pérez-Lloret, S.; Cardinali, D.P. Melatonin as a Chronobiotic and Cytoprotective Agent in Parkinson's Disease. Front. Pharmacol. 2021, 12, 650597. [CrossRef]

59. Ou, B.; Hampsch-Woodill, M.; Prior, R.L. Development and validation of an improved oxygen radical absorbance capacity assay using fluorescein as the fluorescent probe. J. Agric. Food Chem. 2001, 49, 4619-4626. [CrossRef]

60. Di, L.; Kerns, E.H.; Fan, K.; McConnell, O.J.; Carter, G.T. High throughput artificial membrane permeability assay for blood-brain barrier. Eur. J. Med. Chem. 2003, 38, 223-232. [CrossRef]

61. Marzinzig, M.; Nussler, A.K.; Stadler, J.; Marzinzig, E.; Barthlen, W.; Nussler, N.C.; Beger, H.G.; Morris, S.M., Jr.; Brückner, U.B. Improved methods to measure end products of nitric oxide in biological fluids: Nitrite, nitrate, and S-nitrosothiols. Nitric Oxide Biol. Chem. 1997, 1, 177-189. [CrossRef] [PubMed]

62. Itoh, K.; Chiba, T.; Takahashi, S.; Ishii, T.; Igarashi, K.; Katoh, Y.; Oyake, T.; Hayashi, N.; Satoh, K.; Hatayama, I.; et al. An Nrf2/small Maf heterodimer mediates the induction of phase II detoxifying enzyme genes through antioxidant response elements. Biochem. Biophys. Res. Commun. 1997, 236, 313-322. [CrossRef] [PubMed]

63. Halgren, T.A.; Murphy, R.B.; Friesner, R.A.; Beard, H.S.; Frye, L.L.; Pollard, W.T.; Banks, J.L. Glide: A new approach for rapid, accurate docking and scoring. 2. Enrichment factors in database screening. J. Med. Chem. 2004, 47, 1750-1759. [CrossRef]

64. Shelley, J.C.; Cholleti, A.; Frye, L.L.; Greenwood, J.R.; Timlin, M.R.; Uchimaya, M. Epik: A software program for pKa prediction and protonation state generation for drug-like molecules. J. Comput. Aided Mol. Des. 2007, 21, 681-691. [CrossRef] [PubMed]

65. Schrödinger Release 2021-4: LigPrep; Schrödinger, LLC: New York, NY, USA, 2021.

66. Harder, E.; Damm, W.; Maple, J.; Wu, C.; Reboul, M.; Xiang, J.Y.; Wang, L.; Lupyan, D.; Dahlgren, M.K.; Knight, J.L.; et al. OPLS3: A Force Field Providing Broad Coverage of Drug-like Small Molecules and Proteins. J. Chem. Theory Comput. 2016, 12, 281-296. [CrossRef] [PubMed]

67. The PyMOL Molecular Graphics System, Version 2.0; Schrödinger, LLC: New York, NY, USA, 2021.

68. Case, D.A.; Brozell, I.Y.B.-S.S.R.; Cerutti, D.S.; Cheatham, T.E., III; Cruzeiro, V.W.D.; Darden, T.A.; Duke, R.E.; Ghoreishi, D.; Gilson, M.K.; Gohlke, H.; et al. AMBER 2018; University of California: San Francisco, CA, USA, 2018.

69. Jakalian, A.; Jack, D.B.; Bayly, C.I. Fast, efficient generation of high-quality atomic charges. AM1-BCC model: II. Parameterization and validation. J. Comput. Chem. 2002, 23, 1623-1641. [CrossRef]

70. Wang, J.; Wolf, R.M.; Caldwell, J.W.; Kollman, P.A.; Case, D.A. Development and testing of a general amber force field. J. Comput. Chem. 2004, 25, 1157-1174. [CrossRef]

71. Roe, D.R.; Cheatham, T.E., 3rd. PTRAJ and CPPTRAJ: Software for Processing and Analysis of Molecular Dynamics Trajectory Data. J. Chem. Theory Comput. 2013, 9, 3084-3095. [CrossRef]

72. Humphrey, W.; Dalke, A.; Schulten, K. VMD: Visual molecular dynamics. J. Mol. Graph. 1996, 14, 33-38. [CrossRef]

73. Buendia, I.; Navarro, E.; Michalska, P.; Gameiro, I.; Egea, J.; Abril, S.; López, A.; González-Lafuente, L.; López, M.G.; León, R. New melatonin-cinnamate hybrids as multi-target drugs for neurodegenerative diseases: Nrf2-induction, antioxidant effect and neuroprotection. Future Med. Chem. 2015, 7, 1961-1969. [CrossRef]

74. Wang, X.J.; Hayes, J.D.; Wolf, C.R. Generation of a stable antioxidant response element-driven reporter gene cell line and its use to show redox-dependent activation of nrf2 by cancer chemotherapeutic agents. Cancer Res. 2006, 66, 10983-10994. [CrossRef] [PubMed]

75. Magesh, S.; Chen, Y.; Hu, L. Small molecule modulators of Keap1-Nrf2-ARE pathway as potential preventive and therapeutic agents. Med. Res. Rev. 2012, 32, 687-726. [CrossRef] [PubMed]

76. Tripathi, R.K.; Goshain, O.; Ayyannan, S.R. Design, synthesis, in vitro MAO-B inhibitory evaluation, and computational studies of some 6-nitrobenzothiazole-derived semicarbazones. ChemMedChem 2013, 8, 462-474. [CrossRef] [PubMed]

77. Reiter, R.J.; Tan, D.X.; Mayo, J.C.; Sainz, R.M.; Leon, J.; Czarnocki, Z. Melatonin as an antioxidant: Biochemical mechanisms and pathophysiological implications in humans. Acta Biochim. Pol. 2003, 50, 1129-1146. [CrossRef] [PubMed]

78. Gameiro, I.; Michalska, P.; Tenti, G.; Cores, Á.; Buendia, I.; Rojo, A.I.; Georgakopoulos, N.D.; Hernández-Guijo, J.M.; Teresa Ramos, M.; Wells, G.; et al. Discovery of the first dual GSK3 $\beta$ inhibitor/Nrf2 inducer. A new multitarget therapeutic strategy for Alzheimer's disease. Sci. Rep. 2017, 7, 45701. [CrossRef]

79. Hirsch, E.C.; Hunot, S. Neuroinflammation in Parkinson's disease: A target for neuroprotection? Lancet. Neurol. 2009 , 8, $382-397$. [CrossRef]

80. Ransohoff, R.M. How neuroinflammation contributes to neurodegeneration. Science 2016, 353, 777-783. [CrossRef]

81. Liu, T.; Zhang, L.; Joo, D.; Sun, S.C. NF-кB signaling in inflammation. Signal Transduct. Target. Ther. 2017, 2, 17023. [CrossRef] 
82. Cui, W.; Sun, C.; Ma, Y.; Wang, S.; Wang, X.; Zhang, Y. Inhibition of TLR4 Induces M2 Microglial Polarization and Provides Neuroprotection via the NLRP3 Inflammasome in Alzheimer's Disease. Front. Neurosci. 2020, 14, 444. [CrossRef]

83. Gorina, R.; Font-Nieves, M.; Márquez-Kisinousky, L.; Santalucia, T.; Planas, A.M. Astrocyte TLR4 activation induces a proinflammatory environment through the interplay between MyD88-dependent NFkB signaling, MAPK, and Jak1/Stat1 pathways. Glia 2011, 59, 242-255. [CrossRef]

84. Wardyn, J.D.; Ponsford, A.H.; Sanderson, C.M. Dissecting molecular cross-talk between Nrf2 and NF- $\mathrm{B}$ response pathways. Biochem. Soc. Trans. 2015, 43, 621-626. [CrossRef] [PubMed]

85. Lin, W.; Wu, R.T.; Wu, T.; Khor, T.O.; Wang, H.; Kong, A.N. Sulforaphane suppressed LPS-induced inflammation in mouse peritoneal macrophages through Nrf2 dependent pathway. Biochem. Pharmacol. 2008, 76, 967-973. [CrossRef] [PubMed]

86. Michalska, P.; Buendia, I.; Duarte, P.; FernandezMendivil, C.; Negredo, P.; Cuadrado, A.; Lopez, M.G.; Leon, R. Melatoninsulforaphane hybrid ITH12674 attenuates glial response in vivo by blocking LPS binding to MD2 and receptor oligomerization Pharmacol. Res. 2020, 152, 104597. [CrossRef] [PubMed]

87. Dias, V.; Junn, E.; Mouradian, M.M. The role of oxidative stress in Parkinson's disease. J. Parkinson's Dis. 2013, 3, 461-491. [CrossRef]

88. Bonifati, V.; Rizzu, P.; van Baren, M.J.; Schaap, O.; Breedveld, G.J.; Krieger, E.; Dekker, M.C.; Squitieri, F.; Ibanez, P.; Joosse, M.; et al. Mutations in the DJ-1 gene associated with autosomal recessive early-onset parkinsonism. Science 2003, 299, 256-259. [CrossRef]

89. Kapur, A.; Beres, T.; Rathi, K.; Nayak, A.P.; Czarnecki, A.; Felder, M.; Gillette, A.; Ericksen, S.S.; Sampene, E.; Skala, M.C.; et al. Oxidative stress via inhibition of the mitochondrial electron transport and Nrf-2-mediated anti-oxidative response regulate the cytotoxic activity of plumbagin. Sci. Rep. 2018, 8, 1073. [CrossRef]

90. Rodriguez-Pallares, J.; Parga, J.A.; Muñoz, A.; Rey, P.; Guerra, M.J.; Labandeira-Garcia, J.L. Mechanism of 6-hydroxydopamine neurotoxicity: The role of NADPH oxidase and microglial activation in 6-hydroxydopamine-induced degeneration of dopaminergic neurons. J. Neurochem. 2007, 103, 145-156. [CrossRef]

91. Xie, H.R.; Hu, L.S.; Li, G.Y. SH-SY5Y human neuroblastoma cell line: In vitro cell model of dopaminergic neurons in Parkinson's disease. Chin. Med. J. 2010, 123, 1086-1092.

92. Blum, D.; Torch, S.; Lambeng, N.; Nissou, M.; Benabid, A.L.; Sadoul, R.; Verna, J.M. Molecular pathways involved in the neurotoxicity of 6-OHDA, dopamine and MPTP: Contribution to the apoptotic theory in Parkinson's disease. Prog. Neurobiol. 2001, 65, 135-172. [CrossRef]

93. Youdim, M.B.; Gross, A.; Finberg, J.P. Rasagiline [N-propargyl-1R(+)-aminoindan], a selective and potent inhibitor of mitochondrial monoamine oxidase B. Br. J. Pharmacol. 2001, 132, 500-506. [CrossRef]

94. Jiang, H.; Jiang, Q.; Liu, W.; Feng, J. Parkin suppresses the expression of monoamine oxidases. J. Biol. Chem. 2006, 281, 8591-8599. [CrossRef] [PubMed]

95. Hubálek, F.; Binda, C.; Khalil, A.; Li, M.; Mattevi, A.; Castagnoli, N.; Edmondson, D.E. Demonstration of isoleucine 199 as a structural determinant for the selective inhibition of human monoamine oxidase B by specific reversible inhibitors. J. Biol. Chem. 2005, 280, 15761-15766. [CrossRef] [PubMed]

96. Milczek, E.M.; Binda, C.; Rovida, S.; Mattevi, A.; Edmondson, D.E. The 'gating' residues Ile199 and Tyr326 in human monoamine oxidase B function in substrate and inhibitor recognition. FEBS J. 2011, 278, 4860-4869. [CrossRef] [PubMed]

97. Binda, C.; Wang, J.; Pisani, L.; Caccia, C.; Carotti, A.; Salvati, P.; Edmondson, D.E.; Mattevi, A. Structures of human monoamine oxidase B complexes with selective noncovalent inhibitors: Safinamide and coumarin analogs. J. Med. Chem. 2007, 50, 5848-5852. [CrossRef] [PubMed]

98. Gogineni, V.; Nael, M.A.; Chaurasiya, N.D.; Elokely, K.M.; McCurdy, C.R.; Rimoldi, J.M.; Cutler, S.J.; Tekwani, B.L.; León, F. Computationally Assisted Lead Optimization of Novel Potent and Selective MAO-B Inhibitors. Biomedicines 2021, 9, 1304. [CrossRef]

99. Li, M.; Binda, C.; Mattevi, A.; Edmondson, D.E. Functional role of the "aromatic cage" in human monoamine oxidase B: Structures and catalytic properties of Tyr435 mutant proteins. Biochemistry 2006, 45, 4775-4784. [CrossRef]

100. Binda, C.; Li, M.; Hubalek, F.; Restelli, N.; Edmondson, D.E.; Mattevi, A. Insights into the mode of inhibition of human mitochondrial monoamine oxidase B from high-resolution crystal structures. Proc. Natl. Acad. Sci. USA 2003, 100, 9750-9755. [CrossRef]

101. Porritt, M.J.; Kingsbury, A.E.; Hughes, A.J.; Howells, D.W. Striatal dopaminergic neurons are lost with Parkinson's disease progression. Mov. Disord. Off. J. Mov. Disord. Soc. 2006, 21, 2208-2211. [CrossRef]

102. Cunha, M.P.; Martín-de-Saavedra, M.D.; Romero, A.; Egea, J.; Ludka, F.K.; Tasca, C.I.; Farina, M.; Rodrigues, A.L.; López, M.G. Both creatine and its product phosphocreatine reduce oxidative stress and afford neuroprotection in an in vitro Parkinson's model. ASN Neuro 2014, 6, 1759091414554945. [CrossRef]

103. Massari, C.M.; Castro, A.A.; Dal-Cim, T.; Lanznaster, D.; Tasca, C.I. In vitro 6-hydroxydopamine-induced toxicity in striatal, cerebrocortical and hippocampal slices is attenuated by atorvastatin and MK-801. Toxicol. Vitr. Int. J. Publ. Assoc. BIBRA 2016, 37, 162-168. [CrossRef] 
104. Marques, N.F.; Massari, C.M.; Tasca, C.I. Guanosine Protects Striatal Slices Against 6-OHDA-Induced Oxidative Damage, Mitochondrial Dysfunction, and ATP Depletion. Neurotox. Res. 2019, 35, 475-483. [CrossRef] [PubMed]

105. Moldzio, R.; Piskernik, C.; Radad, K.; Rausch, W.D. Rotenone damages striatal organotypic slice culture. Ann. N. Y. Acad. Sci. 2008, 1148, 530-535. [CrossRef] [PubMed] 